\title{
WEAK TENSOR PRODUCTS OF COMPLETE ATOMISTIC LATTICES
}

\author{
BORIS ISCHI
}

\begin{abstract}
Given two complete atomistic lattices $\mathcal{L}_{1}$ and $\mathcal{L}_{2}$, we define a set $\mathrm{S}\left(\mathcal{L}_{1}, \mathcal{L}_{2}\right)$ of complete atomistic lattices by means of three axioms (natural regarding the description of separated quantum compound systems), or in terms of a universal property with respect to a given class of bimorphisms. We call the elements of $\mathrm{S}\left(\mathcal{L}_{1}, \mathcal{L}_{2}\right)$ weak tensor products of $\mathcal{L}_{1}$ and $\mathcal{L}_{2}$. We prove that $\mathrm{S}\left(\mathcal{L}_{1}, \mathcal{L}_{2}\right)$ is a complete lattice. We compare the bottom element $\mathcal{L}_{1} \otimes \mathcal{L}_{2}$ with the separated product of Aerts and with the box product of Grätzer and Wehrung. Similarly, we compare the top element $\mathcal{L}_{1} \otimes \mathcal{L}_{2}$ with the tensor products of Fraser, Chu and Shmuely. With some additional hypotheses on $\mathcal{L}_{1}$ and $\mathcal{L}_{2}$ (true for instance if $\mathcal{L}_{1}$ and $\mathcal{L}_{2}$ are moreover irreducible, orthocomplemented and with the covering property), we characterize the automorphisms of weak tensor products in terms of those of $\mathcal{L}_{1}$ and $\mathcal{L}_{2}$.
\end{abstract}

\section{INTRODUCTION}

In quantum logic, on associates to a physical system $S$ a couple $\left(\Sigma_{S}, \mathcal{L}_{S} \subseteq 2^{\Sigma_{S}}\right)$, where $\Sigma_{S}$ represents the set of all possible states of $S$ and $\mathcal{L}_{S}$, the set of experimental propositions concerning $S$ : A proposition represented by some $a \in \mathcal{L}_{S}$ is true with probability 1 if and only if the state of $S$ lies in $a$ [3. It is usually assumed that $\mathcal{L}_{S}$ is a simple closure space on $\Sigma_{S}$ - in other words, that $\mathcal{L}_{S}$ is closed under arbitrary intersections, and contains $\emptyset, \Sigma_{S}$, and all singletons of $\Sigma_{S}$. In particular, then, $\mathcal{L}_{S}$ is a complete atomistic lattice. Note that any complete atomistic lattice is isomorphic to a simple closure space on its set of atoms.

If $S$ is a compound system consisting of two separated quantum systems $S_{1}$ and $S_{2}$ (for instance two electrons prepared in two different rooms of the lab), then we have [1, 8]

$$
\Sigma_{S}=\Sigma_{S_{1}} \times \Sigma_{S_{2}} .
$$

In particular, simultaneous experiments on both systems can be performed and any experiment done on one system does not alter the state of the other system. Therefore, if $P_{1}$ is a proposition concerning $S_{1}$ represented by some $a_{1} \in \mathcal{L}_{S_{1}}$ and $P_{2}$ is a proposition concerning $S_{2}$ represented by some $a_{2} \in \mathcal{L}_{S_{2}}$, then the proposition $P_{1}$ OR $P_{2}$ concerning the compound system is true with probability 1 if and only if the state $p_{1}$ of $S_{1}$ lies in $a_{1}$ or the state $p_{2}$ of $S_{2}$ lies in $a_{2}$. In other words [1, 8]

$$
a_{1} \times \Sigma_{S_{2}} \cup \Sigma_{S_{1}} \times a_{2} \in \mathcal{L}_{S} \text {, for all } a_{1} \in \mathcal{L}_{S_{1}} \text { and } a_{2} \in \mathcal{L}_{S_{2}} .
$$

2000 Mathematics Subject Classification. Primary 06B23; Secondary 06C15, 81P10. Key words and phrases. Complete atomistic lattice, tensor product, quantum logic. Supported by the Swiss National Science Foundation. 
Moreover, in addition to Axioms $\mathrm{P} 1$ and $\mathrm{P} 2$ above, we postulate that

$$
\text { For all } p_{i} \in \Sigma_{S_{i}} \text { and } \begin{aligned}
A_{i} \subseteq \Sigma_{S_{i}}, & {\left[p_{1} \times A_{2} \in \mathcal{L}_{S} \Rightarrow A_{2} \in \mathcal{L}_{2}\right] \text { and } } \\
& {\left[A_{1} \times p_{2} \in \mathcal{L}_{S} \Rightarrow A_{1} \in \mathcal{L}_{1}\right] . }
\end{aligned}
$$

See [8] for detailed physical justifications of Axioms P1-P3. We define $\mathrm{S}\left(\mathcal{L}_{S_{1}}, \mathcal{L}_{S_{2}}\right)$ to be the set of all simple closure spaces on $\Sigma_{S_{1}} \times \Sigma_{S_{2}}$ for which Axioms P2 and P3 hold.

The rest of the paper is organized as follows. In Section 2, we fix some basic terminology and notation. We define the set $\mathrm{S} \equiv \mathrm{S}\left(\mathcal{L}_{1}, \cdots, \mathcal{L}_{n}\right)$ of $n$-fold weak tensor products. We prove that $S$ is a complete lattice, the bottom and top elements of which are denoted by $\otimes_{i} \mathcal{L}_{i}$ and $\otimes_{i} \mathcal{L}_{i}$ respectively. In Section 3 we compare $₫$ and $\otimes$ with several other tensor products of lattices. An equivalent definition of $S$, in terms of a universal property with respect to a given class of multimorphisms (arbitrary joins are preserved) is given in Section 4. In Section 5 we prove some basic relations between the central elements of $\mathcal{L}_{i}$ 's and those of $\otimes_{\alpha} \mathcal{L}_{\alpha}$ and $\otimes_{\alpha} \mathcal{L}_{\alpha}$.

Let $\mathcal{L} \in \mathrm{S}$ and let $u: \mathcal{L} \rightarrow \mathcal{L}$ preserve arbitrary joins and send atoms to atoms. In Section 6 we prove, under some hypotheses on the image of $u$ and on each $\mathcal{L}_{i}$ (true for instance if $\mathcal{L}_{i}$ is moreover irreducible, orthocomplemented and with the covering property), that there exists a permutation $f$ of $\{1,2, \cdots, n\}$, and joinpreserving maps $v_{i}: \mathcal{L}_{i} \rightarrow \mathcal{L}_{f(i)}$ sending atoms to atoms, such that on atoms $u=$ $f \circ\left(v_{1} \times \cdots \times v_{n}\right)$. A time evolution can be modelled by a map preserving arbitrary joins and sending atoms to atoms 4 . Hence, in the physical interpretation, this result shows that separated quantum systems remain separated only if they do not interact.

\section{MAin DEFinitions}

In this section we give our main definitions. We start with some background material and basic notations used in the sequel.

Definition 2.1. Let $\Sigma$ be a non-empty set and $\omega$ a set of subsets of $\Sigma$. We write $\bigcap \omega$ (respectively $\bigcup \omega$ ) instead of $\bigcap_{a \in \omega} a$ (respectively $\bigcup_{a \in \omega} a$ ). By a simple closure space $\mathcal{L}$ on $\Sigma$ we mean a set of subsets of $\Sigma$, ordered by set-inclusion, closed under arbitrary set-intersections (i.e., for all $\omega \subseteq \mathcal{L}, \bigcap \omega \in \mathcal{L}$ ), and containing $\Sigma$, $\emptyset$, and all singletons. We denote the bottom $(\emptyset)$ and top $(\Sigma)$ elements by 0 and 1 respectively. For $p \in \Sigma$, we identify $p$ with $\{p\} \in \mathcal{L}$. Hence $p \cup q$ stands for $\{p, q\}$.

We denote the category of simple closure spaces with maps preserving arbitrary joins (hence 0 ) by $\mathbf{C l}$, and the sub-category of simple closure spaces on a particular (nonempty) set $\Sigma$, by $\mathbf{C l}(\Sigma)$. Let 2 denote the simple closure space isomorphic to the two-element lattice.

Let $\mathcal{L}, \mathcal{L}_{1} \in \mathbf{C l}(\Sigma)$ and $u: \mathcal{L} \rightarrow \mathcal{L}_{1}$ a map sending atoms to atoms. We write $\operatorname{Aut}(\mathcal{L})$ for the group of automorphisms of $\mathcal{L}$ and we also call $u$ the mapping from $\Sigma$ to $\Sigma_{1}$ induced by $u$.

Remark 2.2. Let $\mathcal{L}$ be a simple closure space on a (nonempty) set $\Sigma$. Then $\mathcal{L}$ is a complete atomistic lattice, the atoms of which correspond to the points (i.e., singletons) of $\Sigma$. Note that if $A \subseteq \Sigma$, then $\bigvee_{\mathcal{L}}(A)=\bigcap\{b \in \mathcal{L} ; A \subseteq b\}$.

Conversely, let $\mathcal{L}$ be a complete atomistic lattice. Let $\Sigma$ denote the set of atoms of $\mathcal{L}$, and, for each $a \in \mathcal{L}$, let $\Sigma[a]$ denote the set of atoms under $a$. Then $\{\Sigma[a] ; a \in \mathcal{L}\}$ is a simple closure space on $\Sigma$, isomorphic to $\mathcal{L}$. 
Finally, let $\mathcal{L}$ be a simple closure space and let $u \in \operatorname{Aut}(\mathcal{L})$. Then, note that for all $a \in \mathcal{L}, u(a)=\{u(p) ; p \in a\}$.

Definition 2.3. Let $\left\{\Sigma_{\alpha}\right\}_{\alpha \in \Omega}$ be a family of nonempty sets, $\boldsymbol{\Sigma}=\prod_{\alpha} \Sigma_{\alpha}, p \in \boldsymbol{\Sigma}$, $R \subseteq \boldsymbol{\Sigma}, A \in \prod_{\alpha} 2^{\Sigma_{\alpha}}, \beta \in \Omega$, and $B \subseteq \Sigma_{\beta}$. We shall make use of the following notations:

(1) We denote by $\pi_{\beta}: \Sigma \rightarrow \Sigma_{\beta}$ the $\beta$-th coordinate map, i.e., $\pi_{\beta}(p)=p_{\beta}$.

(2) We denote by $p[-, \beta]: \Sigma_{\beta} \rightarrow \boldsymbol{\Sigma}$ the map that sends $q \in \Sigma_{\beta}$ to the element of $\boldsymbol{\Sigma}$ obtained by replacing $p$ 's $\beta$-th entry by $q$.

(3) We define $R_{\beta}[p]=\pi_{\beta}\left(p\left[\Sigma_{\beta}, \beta\right] \cap R\right)$. Note that $R_{\beta}[p]=\left\{q \in \Sigma_{\beta} ; p[q, \beta] \in\right.$ $R\}$.

(4) We define $A[B, \beta] \in \prod_{\alpha} 2^{\Sigma_{\alpha}}$ as $A[B, \beta]_{\beta}=B$ and $A[B, \beta]_{\alpha}=A_{\alpha}$ for $\alpha \neq \beta$.

(5) We write $\bar{A}:=\prod_{\alpha} A_{\alpha}$ and $\bar{A}[B, \beta]:=\overline{A[B, \beta]}$.

We omit the $\beta$ in $p[-, \beta]$ when no confusion can occur. For instance, we write $p\left[\Sigma_{\beta}\right]$ instead of $p\left[\Sigma_{\beta}, \beta\right]$.

Remark 2.4. $p\left[R_{\beta}[p]\right]=p\left[\Sigma_{\beta}\right] \cap R$.

Definition 2.5. Let $\left\{\mathcal{L}_{\alpha}\right\}_{\alpha \in \Omega}$ be a family of simple closure spaces on $\Sigma_{\alpha}$. We denote by $\mathrm{S}\left(\mathcal{L}_{\alpha}, \alpha \in \Omega\right)$ the set all $\mathcal{L} \in \mathbf{C l}(\boldsymbol{\Sigma})$ such that

(P1) $\boldsymbol{\Sigma}=\prod_{\alpha} \Sigma_{\alpha}$

(P2) $\bigcup_{\alpha} \pi_{\alpha}^{-1}\left(a_{\alpha}\right) \in \mathcal{L}$, for all $a \in \prod_{\alpha} \mathcal{L}_{\alpha}$,

(P3) for all $p \in \boldsymbol{\Sigma}, \beta \in \Omega$, and $B \subseteq \Sigma_{\beta},\left[p[B, \beta] \in \mathcal{L} \Rightarrow B \in \mathcal{L}_{\beta}\right]$.

Let $T=\prod_{\alpha} T_{\alpha}$ with $T_{\alpha} \subseteq \operatorname{Aut}\left(\mathcal{L}_{\alpha}\right)$. We denote by $\mathcal{S}_{T}\left(\mathcal{L}_{\alpha}, \alpha \in \Omega\right)$ the set of all $\mathcal{L} \in \mathrm{S}\left(\mathcal{L}_{\alpha}, \alpha \in \Omega\right)$ such that

(P4) for all $v \in T$, there is $u \in \operatorname{Aut}(\mathcal{L})$ such that $u(p)_{\alpha}=v_{\alpha}\left(p_{\alpha}\right)$ for all $p \in \boldsymbol{\Sigma}$ and all $\alpha \in \Omega$.

We call elements of $\mathrm{S}\left(\mathcal{L}_{\alpha}, \alpha \in \Omega\right)$ weak tensor products of the family $\left\{\mathcal{L}_{\alpha}\right\}_{\alpha \in \Omega}$.

Remark 2.6. The $u$ in Axiom $\mathrm{P} 4$ is necessarily unique. Note also that for all $T=\prod_{\alpha} T_{\alpha}$ with $T_{\alpha} \subseteq \operatorname{Aut}\left(\mathcal{L}_{\alpha}\right), \mathcal{S}_{T}\left(\mathcal{L}_{\alpha}, \alpha \in \Omega\right) \subseteq \mathrm{S}\left(\mathcal{L}_{\alpha}, \alpha \in \Omega\right)$. The name "weak tensor product" is justified by the fact that $\mathrm{S}\left(\overline{\mathcal{L}}_{\alpha}, \alpha \in \Omega\right)$ and $\mathcal{S}_{T}\left(\mathcal{L}_{\alpha}, \alpha \in \Omega\right)$ can be defined in terms of a universal property with respect to a given class of multimorphisms of $\mathbf{C l}$ (see Section 4).

Lemma 2.7. Let $\left\{\mathcal{L}_{\alpha}\right\}_{\alpha \in \Omega}$ be a family of simple closure spaces on $\Sigma_{\alpha}, \beta \in \Omega$, and $\mathcal{L} \in \mathrm{S}\left(\mathcal{L}_{\alpha}, \alpha \in \Omega\right)$.

(1) For any $a \in \prod_{\alpha} \mathcal{L}_{\alpha}, \bar{a} \in \mathcal{L}$.

(2) For any $b \in \mathcal{L}_{\beta}$ and every $p \in \prod_{\alpha} \Sigma_{\alpha}, p[b, \beta] \in \mathcal{L}$.

(3) For any $B \subseteq \mathcal{L}_{\beta}$ and every $a \in \prod_{\alpha} \mathcal{L}_{\alpha}, \bar{a}[\bigvee B, \beta]=\bigvee_{b \in B} \bar{a}[b, \beta]$

Proof. (1) Let $\beta \in \Omega$. Define $\widehat{a}^{\beta} \in \prod_{\alpha} \mathcal{L}_{\alpha}$ by setting $\widehat{a}_{\alpha}^{\beta}=a_{\beta}$ if $\alpha=\beta$, and $\emptyset$ otherwise. Note that $\pi_{\beta}^{-1}\left(a_{\beta}\right)=\bigcup_{\alpha} \pi_{\alpha}^{-1}\left(\widehat{a}_{\alpha}^{\beta}\right)$. Now, by Axiom P2, $\bigcup_{\alpha} \pi_{\alpha}^{-1}\left(\widehat{a}_{\alpha}^{\beta}\right) \in \mathcal{L}$, therefore $\pi_{\beta}^{-1}\left(a_{\beta}\right) \in \mathcal{L}$. Finally $\bar{a}=\bigcap_{\alpha} \pi_{\alpha}^{-1}\left(a_{\alpha}\right)$, hence $\bar{a} \in \mathcal{L}$.

(2) Define $a \in \prod_{\alpha} \mathcal{L}_{\alpha}$ as $a_{\alpha}=p_{\alpha}$ if $\alpha \neq \beta$ and $a_{\beta}=b$. Then $p[b, \beta]=\prod_{\alpha} a_{\alpha}$, hence from the first part $p[b, \beta] \in \mathcal{L}$.

(3) By the first part, $\bar{a}[\bigvee B, \beta] \in \mathcal{L}$. Moreover, $\bar{a}[b, \beta] \subseteq \bar{a}[\bigvee B, \beta]$ for all $b \in B$, hence $\bigvee_{b \in B} \bar{a}[b, \beta] \subseteq \bar{a}[\bigvee B, \beta]$. As a consequence, there is $X \subseteq \Sigma_{\beta}$ such that $\bigvee_{b \in B} \bar{a}[b, \beta]=\bar{a}[X, \beta]$ and $X \subseteq \bigvee B$. Moreover, $\bar{a}[X, \beta] \in \mathcal{L}$, therefore $p[X, \beta] \in \mathcal{L}$ for any $p \in \bar{a}$, whence, by Axiom P3, it follows that $X \in \mathcal{L}_{\beta}$. Finally, $B \subseteq X$, therefore $\bigvee B \subseteq X$. As a consequence, $X=\bigvee B$. 
Lemma 2.8. Let $\left\{\mathcal{L}_{\alpha}\right\}_{\alpha \in \Omega}$ be a family of simple closure spaces on $\Sigma_{\alpha}, \boldsymbol{\Sigma}=\prod_{\alpha} \Sigma_{\alpha}$, and $\mathcal{L}_{0}, \mathcal{L}$ and $\mathcal{L}_{1}$ be simple closures spaces on $\boldsymbol{\Sigma}$. Suppose that $\mathcal{L}_{0} \subseteq \mathcal{L} \subseteq \mathcal{L}_{1}$.

(1) If Axiom P2 holds in $\mathcal{L}_{0}$, then it holds also in $\mathcal{L}$.

(2) If Axiom P3 holds in $\mathcal{L}_{1}$, then it holds also in $\mathcal{L}$.

Proof. Direct from Definition 2.5

Definition 2.9. Let $\left\{\Sigma_{\alpha}\right\}_{\alpha \in \Omega}$ be a family of nonempty sets and $\left\{\mathcal{L}_{\alpha} \subseteq 2^{\Sigma_{\alpha}}\right\}_{\alpha \in \Omega}$. Let $\boldsymbol{\Sigma}=\prod_{\alpha} \Sigma_{\alpha}$. Then

$$
\begin{aligned}
& \otimes\left\{\mathcal{L}_{\alpha} ; \alpha \in \Omega\right\}:=\left\{\bigcap \omega ; \omega \subseteq\left\{\bigcup_{\alpha} \pi_{\alpha}^{-1}\left(a_{\alpha}\right) ; a \in \prod_{\alpha} \mathcal{L}_{\alpha}\right\}\right\}, \\
& \otimes\left\{\mathcal{L}_{\alpha} ; \alpha \in \Omega\right\}:=\left\{R \subseteq \boldsymbol{\Sigma} ; R_{\beta}[p] \in \mathcal{L}_{\beta}, \forall p \in \boldsymbol{\Sigma}, \beta \in \Omega\right\},
\end{aligned}
$$

ordered by set-inclusion.

Lemma 2.10. Let $\left\{\mathcal{L}_{\alpha}\right\}_{\alpha \in \Omega}$ be a family of simple closure spaces on $\Sigma_{\alpha}$ with $\boldsymbol{\Sigma}=$ $\prod_{\alpha} \Sigma_{\alpha}$, and let $\mathcal{L}_{0}$ and $\mathcal{L}_{1}$ be simple closures spaces on $\boldsymbol{\Sigma}$.

(1) If Axiom P2 holds in $\mathcal{L}_{0}$, then $\otimes_{\alpha} \mathcal{L}_{\alpha} \subseteq \mathcal{L}_{0}$.

(2) If Axiom P3 holds in $\mathcal{L}_{1}$ and if $p\left[\Sigma_{\beta}, \beta\right] \in \mathcal{L}_{1}$, for all $p \in \boldsymbol{\Sigma}$ and all $\beta \in \Omega$, then $\mathcal{L}_{1} \subseteq \otimes_{\alpha} \mathcal{L}_{\alpha}$.

Proof. (1) Direct from Definitions 2.5 and 2.9 .

(2) Let $R \in \mathcal{L}_{1}, p \in \boldsymbol{\Sigma}$, and $\beta \in \Omega$. By hypothesis, $p\left[\Sigma_{\beta}, \beta\right] \in \mathcal{L}_{1}$, hence $p\left[\Sigma_{\beta}, \beta\right] \cap R \in \mathcal{L}_{1}$. Now, $p\left[\Sigma_{\beta}, \beta\right] \cap R=p\left[R_{\beta}[p]\right]$ (see Remark 2.4). As a consequence, $p\left[R_{\beta}[p]\right] \in \mathcal{L}_{1}$ therefore, by Axiom P3, $R_{\beta}[p] \in \mathcal{L}_{\beta}$.

Proposition 2.11. Let $\left\{\Sigma_{\alpha}\right\}_{\alpha \in \Omega}$ be a family of nonempty sets, and let $\left\{\mathcal{L}_{\alpha} \subseteq\right.$ $\left.2^{\Sigma_{\alpha}}\right\}_{\alpha \in \Omega}$. Let $\left\{\Omega_{\gamma} \subseteq \Omega ; \gamma \in \Gamma\right\}$ such that $\Omega=\coprod\left\{\Omega_{\gamma} ; \gamma \in \Gamma\right\}$. Then

$$
\begin{aligned}
& \otimes\left\{\mathcal{L}_{\alpha} ; \alpha \in \Omega\right\}=\bigotimes_{\gamma \in \Gamma}\left(\bigotimes_{\alpha \in \Omega_{\gamma}} \mathcal{L}_{\alpha}\right), \\
& \bigotimes\left\{\mathcal{L}_{\alpha} ; \alpha \in \Omega\right\}=\bigotimes_{\gamma \in \Gamma}\left(\bigotimes_{\alpha \in \Omega_{\gamma}} \mathcal{L}_{\alpha}\right) .
\end{aligned}
$$

Proof. Direct from Definition 2.9.

Theorem 2.12. Let $\left\{\mathcal{L}_{\alpha}\right\}_{\alpha \in \Omega}$ be a family of simple closure spaces on $\Sigma_{\alpha}, \boldsymbol{\Sigma}=$ $\prod_{\alpha} \Sigma_{\alpha}$, and $T=\prod_{\alpha} T_{\alpha}$ with $T_{\alpha} \subseteq \operatorname{Aut}\left(\mathcal{L}_{\alpha}\right)$.

(1) $\otimes_{\alpha} \mathcal{L}_{\alpha}$ and $\otimes_{\alpha} \mathcal{L}_{\alpha}$ are simple closure spaces on $\boldsymbol{\Sigma}$.

(2) $\otimes_{\alpha} \mathcal{L}_{\alpha}, \otimes_{\alpha} \mathcal{L}_{\alpha} \in \mathcal{S}_{T}\left(\mathcal{L}_{\alpha}, \alpha \in \Omega\right)$

(3) $\mathrm{S}\left(\mathcal{L}_{\alpha}, \alpha \in \Omega\right)=\left\{\mathcal{L} \in \mathbf{C l}(\boldsymbol{\Sigma}) ; \otimes_{\alpha} \mathcal{L}_{\alpha} \subseteq \mathcal{L} \subseteq \otimes_{\alpha} \mathcal{L}_{\alpha}\right\}$

(4) $\mathcal{S}_{T}\left(\mathcal{L}_{\alpha}, \alpha \in \Omega\right)$ and $\mathrm{S}\left(\mathcal{L}_{\alpha}, \alpha \in \Omega\right)$, ordered by set-inclusion, are complete lattices.

Proof. (1) Obviously, $\otimes_{\alpha} \mathcal{L}_{\alpha}$ and $\otimes_{\alpha} \mathcal{L}_{\alpha}$ contain $\emptyset$ and $\boldsymbol{\Sigma}$, and by definition $\otimes_{\alpha} \mathcal{L}_{\alpha}$ is $\cap$-closed and $\otimes_{\alpha} \mathcal{L}_{\alpha}$ contains all singletons of $\boldsymbol{\Sigma}$. If $\omega \subseteq \bigotimes_{\alpha} \mathcal{L}_{\alpha}$, then $(\bigcap \omega)_{\beta}[p]=$ $\bigcap\left\{R_{\beta}[p] ; R \in \omega\right\}$. Moreover, for all $p \in \Sigma, \bigcap_{\alpha} \pi_{\alpha}^{-1}\left(p_{\alpha}\right)=p$. As a consequence, $\otimes_{\alpha} \mathcal{L}_{\alpha}$ and $\otimes_{\alpha} \mathcal{L}_{\alpha}$ are simple closure spaces on $\boldsymbol{\Sigma}$.

(2) By definition, Axiom P2 holds in $\otimes_{\alpha} \mathcal{L}_{\alpha}$ and Axiom P3 holds in $\bigotimes_{\alpha} \mathcal{L}_{\alpha}$.

Let $a \in \prod_{\alpha} \mathcal{L}_{\alpha}, \beta \in \Omega, p \in \boldsymbol{\Sigma}$, and $R=\bigcup_{\alpha} \pi_{\alpha}^{-1}\left(a_{\alpha}\right)$. Then $R_{\beta}[p]=\Sigma_{\beta}$ if $p_{\alpha} \in a_{\alpha}$ for some $\alpha \neq \beta$, and $R_{\beta}[p]=a_{\beta}$ otherwise. As a consequence, $\bigcup_{\alpha} \pi_{\alpha}^{-1}\left(a_{\alpha}\right) \in \bigotimes_{\alpha} \mathcal{L}_{\alpha}$, hence Axiom P2 holds in $\oslash_{\alpha} \mathcal{L}_{\alpha}$, therefore $\bigotimes_{\alpha} \mathcal{L}_{\alpha} \in \mathrm{S}\left(\mathcal{L}_{\alpha}, \alpha \in \Omega\right)$.

By Lemma 2.10 part (1), $\otimes_{\alpha} \mathcal{L}_{\alpha} \subseteq \bigotimes_{\alpha} \mathcal{L}_{\alpha}$, hence by Lemma 2.8, Axiom P3 holds in $\otimes_{\alpha} \mathcal{L}_{\alpha}$. As a consequence, $\otimes_{\alpha} \mathcal{L}_{\alpha} \in \mathrm{S}\left(\mathcal{L}_{\alpha}, \alpha \in \Omega\right)$. 
Finally, let $v \in T, a \in \prod_{\alpha} \mathcal{L}_{\alpha}, p \in \boldsymbol{\Sigma}, R \subseteq \boldsymbol{\Sigma}$, and $\omega \subseteq 2^{\boldsymbol{\Sigma}}$. Then,

$$
v\left(\bigcup_{\alpha} \pi_{\alpha}^{-1}\left(a_{\alpha}\right)\right)=\bigcup_{\alpha} \pi_{\alpha}^{-1}\left(v_{\alpha}\left(a_{\alpha}\right)\right),
$$

and

$$
v(R)_{\beta}[v(p)]=v_{\beta}\left(R_{\beta}[p]\right)
$$

Moreover $v(\bigcap \omega)=\bigcap\{v(x) ; x \in \omega\}$. Hence, $v$ is a bijection of $\otimes_{\alpha} \mathcal{L}_{\alpha}$ and of $\oslash_{\alpha} \mathcal{L}_{\alpha}$, and $v$ preserves arbitrary meets, hence also arbitrary joins.

(3) Follows directly from Lemmata 2.8 and 2.10.

(4) Let $\omega \subseteq \mathrm{S}\left(\mathcal{L}_{\alpha}, \alpha \in \Omega\right)$ (respectively $\omega \subseteq \mathcal{S}_{T}\left(\mathcal{L}_{\alpha}, \alpha \in \Omega\right)$ ). Then obviously $\bigcap \omega=\{a \in \mathcal{L}, \forall \mathcal{L} \in \omega\} \in \mathrm{S}\left(\mathcal{L}_{\alpha}, \alpha \in \Omega\right)$ (respectively $\bigcap \omega \in \mathcal{S}_{T}\left(\mathcal{L}_{\alpha}, \alpha \in \Omega\right)$ ).

Remark 2.13. Let $\left\{\mathcal{L}_{\alpha}\right\}_{\alpha \in \Omega}$ be a family of simple closure spaces on $\Sigma_{\alpha}$. For all $T=\prod_{\alpha} T_{\alpha}$ with $T_{\alpha} \subseteq \operatorname{Aut}\left(\mathcal{L}_{\alpha}\right), \mathcal{S}_{T}\left(\mathcal{L}_{\alpha}, \alpha \in \Omega\right)$ is a complete meet-sublattice of $\mathrm{S}\left(\mathcal{L}_{\alpha}, \alpha \in \Omega\right)$. Moreover, $\otimes_{\alpha} \mathcal{L}_{\alpha}$ and $\oslash_{\alpha} \mathcal{L}_{\alpha}$ are the bottom and the top elements of $\mathrm{S}\left(\mathcal{L}_{\alpha}, \alpha \in \Omega\right)$ respectively.

Proposition 2.14. Let $\mathcal{L}_{1}$ and $\mathcal{L}_{2}$ be simple closure spaces. Then $\mathcal{L}_{1} \otimes \mathcal{L}_{2} \cong$ $\mathcal{L}_{2} \otimes \mathcal{L}_{1}, \mathcal{L}_{1} \otimes \mathcal{L}_{2} \cong \mathcal{L}_{2} \otimes \mathcal{L}_{1}$, and $2 \otimes \mathcal{L}_{1} \cong \mathcal{L}_{1}$

Proof. Direct from Definition 2.9.

More generally, then, we have:

Proposition 2.15. Let $\left\{\mathcal{L}_{\alpha}\right\}_{\alpha \in \Omega}$ be a family of simple closure spaces on $\Sigma_{\alpha}$, and $T=\prod_{\alpha} T_{\alpha}$ with $T_{\alpha} \subseteq$ Aut $\left(\mathcal{L}_{\alpha}\right)$. Then there is an isomorphism $f: \mathcal{S}_{T}\left(\mathcal{L}_{\alpha}, \alpha \in\right.$ $\Omega) \rightarrow \mathcal{S}_{T}\left(2, \mathcal{L}_{\alpha}, \alpha \in \Omega\right)$ such that for all $\mathcal{L} \in \mathcal{S}_{T}\left(\mathcal{L}_{\alpha}, \alpha \in \Omega\right), \mathcal{L} \cong f(\mathcal{L})$.

Proof. Direct from Definition 2.5 .

Example 2.16. We now consider, for contrast, two examples, well known in manybody quantum physics, where instead of Axioms P1 and P2, we have

(p1) $\exists f: \prod_{\alpha} \Sigma_{\alpha} \rightarrow \boldsymbol{\Sigma}$ with $f$ injective,

(p2) $f\left(\prod_{\alpha} \Sigma_{\alpha}\right) \cap\left(\bigvee f\left(\bigcup_{\alpha} \pi_{\alpha}^{-1}\left(a_{\alpha}\right)\right)\right)=f\left(\bigcup_{\alpha} \pi_{\alpha}^{-1}\left(a_{\alpha}\right)\right), \forall a \in \prod_{\alpha} \mathcal{L}_{\alpha}$.

If $\mathcal{H}$ is a complex Hilbert space, then $\Sigma_{\mathcal{H}}$ denotes the set of one-dimensional subspaces of $\mathcal{H}$ and $\mathrm{P}(\mathcal{H})$ stands for the simple closure space isomorphic to the lattice of closed subspaces of $\mathcal{H}$. Moreover, we write $\mathrm{U}(\mathcal{H})$ for the group of automorphisms of $\mathrm{P}(\mathcal{H})$ induced by unitary maps on $\mathcal{H}$.

Let $\mathcal{H}_{1}$ and $\mathcal{H}_{2}$ be complex Hilbert spaces, $\mathcal{L}_{1}=\mathrm{P}\left(\mathcal{H}_{1}\right), \mathcal{L}_{2}=\mathrm{P}\left(\mathcal{H}_{2}\right)$ and $\mathcal{L}=\mathrm{P}\left(\mathcal{H}_{1} \otimes \mathcal{H}_{2}\right)$. Then, Axioms p1 (with $\boldsymbol{\Sigma}=\Sigma_{\mathcal{H}_{1} \otimes \mathcal{H}_{2}}$ ), p2 and p3 (replace $p[B, \beta]$ by $f(p[B, \beta])$ in Axiom P3) hold in $\mathcal{L}$. Moreover, Axiom p4 (replace $u(p)_{\alpha}=v_{\alpha}\left(p_{\alpha}\right)$ by $f^{-1}(u(f(p)))_{\alpha}=v_{\alpha}\left(p_{\alpha}\right)$ in Axiom P4) holds for $T=\mathrm{U}\left(\mathcal{H}_{1}\right) \times \mathrm{U}\left(\mathcal{H}_{2}\right)$. Note that $\bigvee\left(A_{1} \times A_{2}\right)=1$, for all $A_{i} \subseteq \Sigma_{\mathcal{H}_{i}}$ with $\bigvee A_{i}=1$

Let $\mathcal{H}$ be a complex Hilbert space and $\mathcal{F}=\bigoplus_{n>0} \mathcal{H}^{\otimes n}$ be the Fock space (neither symmetrized, nor antisymmetrized). Let $\mathcal{L}=\mathrm{P}(\mathcal{F})$, and for all integer $i$, let $\mathcal{L}_{i}=\mathrm{P}(\mathcal{H})$. Let $n$ be an integer and consider the family $\left\{\mathcal{L}_{i} ; 1 \leq i \leq n\right\}$. Then Axioms p1, p2, p3 and p4 with $T_{i}=\mathrm{U}(\mathcal{H})$ hold in $\mathcal{L}$ for all $n$. For all $i$, let $\Sigma_{i}=\Sigma_{\mathcal{H}}$. Note that in that case, for all $n$ we have $\bigvee\left(\prod_{i=1}^{n} \Sigma_{i}\right) \neq 1$ 


\section{Comparison With Other TENSOR PRODUCTS}

In this section, we compare the bottom element $\mathcal{L}_{1} \otimes \mathcal{L}_{2}$ with the separated product of Aerts and with the box product $\mathcal{L}_{1} \square \mathcal{L}_{2}$ of Grätzer and Wehrung. On the other hand, we compare the top element $\mathcal{L}_{1} \otimes \mathcal{L}_{2}$ with the semilattice tensor product of Fraser and with the tensor products of Chu and Shmuely.

\subsection{The separated product.}

Definition 3.1. A lattice $\mathcal{L}$ with 0 and 1 is orthocomplemented if there is a unary operation ${ }^{\perp}: \mathcal{L} \rightarrow \mathcal{L}$ such that for all $a, b \in \mathcal{L},\left(a^{\perp}\right)^{\perp}=a, a \leq b$ implies $b^{\perp} \leq a^{\perp}$, and $a \bigwedge a^{\perp}=0$.

Let $\mathcal{L}$ be an orthocomplemented simple closure space on $\Sigma$. Then, for $p, q \in \Sigma$, we write $p \perp q$ if $p \in q^{\perp}$, where $q^{\perp}$ stands for $\{q\}^{\perp}$.

Remark 3.2. Note that the binary relation $\perp$ on $\Sigma$ is symmetric, anti-reflexive and separating, i.e., for all $p \neq q \in \Sigma$, there is $r \in \Sigma$ such that $p \perp r$ and $q \not \perp r$.

Conversely, let $\Sigma$ be a set and $\perp$ a symmetric, anti-reflexive and separating binary relation on $\Sigma$. Then $\mathcal{L}=\left\{A \subseteq \Sigma ; A^{\perp \perp}=A\right\}$ is an orthocomplemented simple closure space on $\Sigma$.

Definition 3.3 (D. Aerts, [1]). Let $\left\{\mathcal{L}_{\alpha}\right\}_{\alpha \in \Omega}$ be a family of orthocomplemented simple closure spaces on $\Sigma_{\alpha}, \boldsymbol{\Sigma}=\prod_{\alpha} \Sigma_{\alpha}$, and let $p, q \in \boldsymbol{\Sigma}$. Denote by \# the binary relation on $\boldsymbol{\Sigma}$ defined by $p \# q$ if and only if there is $\beta \in \Omega$ such that $p_{\beta} \perp_{\beta} q_{\beta}$. Then

$$
\underset{\operatorname{Aerts}_{\alpha}}{\otimes} \mathcal{L}_{\alpha}:=\left\{R \subseteq \boldsymbol{\Sigma} ; R^{\# \#}=R\right\}
$$

Lemma 3.4. Let $\left\{\mathcal{L}_{\alpha}\right\}_{\alpha \in \Omega}$ be a family of orthocomplemented simple closure spaces on $\Sigma_{\alpha}$. Then

and $\underset{\operatorname{Aerts}_{\alpha}}{\otimes} \mathcal{L}_{\alpha}$ is orthocomplemented.

$$
\underset{\operatorname{Aerts}_{\alpha}}{\otimes} \mathcal{L}_{\alpha}=\otimes_{\alpha} \mathcal{L}_{\alpha}
$$

Proof. Let $\boldsymbol{\Sigma}=\prod_{\alpha} \Sigma_{\alpha}$. Obviously, \# is symmetric and anti-reflexive. Since $\mathcal{L}_{\alpha}$ is orthocomplemented, $\perp_{\alpha}$ is separating. Therefore, it follows directly from Definition 3.3 that \# is also separating. As a consequence, $\underset{\operatorname{Aerts}_{\alpha}}{\otimes} \mathcal{L}_{\alpha}$ is an orthocomplemented simple closure space on $\boldsymbol{\Sigma}$. Moreover, coatoms are given by $p^{\#}=\bigcup_{\alpha} \pi_{\alpha}^{-1}\left(p_{\alpha}^{\perp_{\alpha}}\right)$. As a consequence, $\underset{\text { Aerts } \alpha}{\otimes} \mathcal{L}_{\alpha} \subseteq \bigotimes_{\alpha} \mathcal{L}_{\alpha}$.

Let $a \in \prod_{\alpha} \mathcal{L}_{\alpha}$. Denote the set of coatoms of $\mathcal{L}_{\alpha}$ above $a_{\alpha}$ by $\Sigma^{\prime}\left[a_{\alpha}\right]$. Then $a_{\alpha}=\bigcap_{\alpha} \Sigma^{\prime}\left[a_{\alpha}\right]$. Moreover,

$$
\bigcap\left\{\bigcup_{\alpha} \pi_{\alpha}^{-1}\left(x_{\alpha}\right) ; x \in \prod_{\alpha} \Sigma^{\prime}\left[a_{\alpha}\right]\right\}=\bigcup_{\alpha} \pi_{\alpha}^{-1}\left(a_{\alpha}\right) .
$$

Hence $\bigcup_{\alpha} \pi_{\alpha}^{-1}\left(a_{\alpha}\right) \in \underset{\operatorname{Aerts}_{\alpha}}{\otimes} \mathcal{L}_{\alpha}$. Another way to see this is to compute

$$
\left(\bigcup_{\alpha} \pi_{\alpha}^{-1}\left(a_{\alpha}\right)\right)^{\#}=\bigcap_{\alpha}\left(\pi_{\alpha}^{-1}\left(a_{\alpha}\right)\right)^{\#}=\bigcap_{\alpha} \pi_{\alpha}^{-1}\left(a_{\alpha}^{\perp_{\alpha}}\right)=\prod_{\alpha} a_{\alpha}^{\perp_{\alpha}},
$$

whence

$$
\left(\bigcup_{\alpha} \pi_{\alpha}^{-1}\left(a_{\alpha}\right)\right)^{\# \#}=\bigcup_{\alpha} \pi_{\alpha}^{-1}\left(a_{\alpha}^{\perp_{\alpha} \perp_{\alpha}}\right)=\bigcup_{\alpha} \pi_{\alpha}^{-1}\left(a_{\alpha}\right) .
$$


As a consequence, $\oslash_{\alpha} \mathcal{L}_{\alpha} \subseteq \underset{\operatorname{Aerts}_{\alpha}}{\otimes} \mathcal{L}_{\alpha}$.

Remark 3.5. The symbol $₫$ was originally used by Aerts.

\subsection{The box product.}

Definition 3.6. Let $\mathcal{L}$ be a lattice and $a \in \mathcal{L}$. We denote by $a \downarrow$ the set $\{x \in$ $\mathcal{L} ; x \leq a\}$ and by $L$ the set $\{a \downarrow ; a \in \mathcal{L}\}$ ordered by set-inclusion (note that $\left.L \subseteq 2^{\overline{\mathcal{L}}}\right)$

Definition 3.7 (G. Grätzer, F. Wehrung, [7]). Let $\mathcal{L}_{1}$ and $\mathcal{L}_{2}$ be lattices and $(a, b) \in \mathcal{L}_{1} \times \mathcal{L}_{2}$. Define

$$
a \square b=\left(a \downarrow \times \mathcal{L}_{2}\right) \cup\left(\mathcal{L}_{1} \times b \downarrow\right) .
$$

The box product $\mathcal{L}_{1} \square \mathcal{L}_{2}$ is defined as the set of all finite intersections of the form $\bigcap\left\{a_{i} \square b_{i} ; i \leq n\right\}$ where $\left(a_{i}, b_{i}\right) \in \mathcal{L}_{1} \times \mathcal{L}_{2}$ for all $i \leq n$, ordered by set-inclusion.

Remark 3.8. Obviously, $\mathcal{L}_{1} \square \mathcal{L}_{2}$ is a meet-sublattice of $2^{\mathcal{L}_{1} \times \mathcal{L}_{2}}$. In fact, $\mathcal{L}_{1} \square \mathcal{L}_{2}$ is a lattice (see [7, Proposition 2.9). Note that if $\mathcal{L}_{1}$ and $\mathcal{L}_{2}$ have top elements, then $\mathcal{L}_{1} \otimes \mathcal{L}_{2}=\mathcal{L}_{1} \square \mathcal{L}_{2}$ where $\mathcal{L}_{1} \otimes \mathcal{L}_{2}$ is the lattice tensor product (see [7]).

Definition 3.9. Let $\Sigma_{1}$ and $\Sigma_{2}$ be nonempty sets, $\mathcal{L}_{1} \subseteq 2^{\Sigma_{1}}$ and $\mathcal{L}_{2} \subseteq 2^{\Sigma_{2}}$. Define $\mathcal{L}_{1} \oslash_{n} \mathcal{L}_{2}$ as in Definition 2.9 but taking only finite intersections. If $\mathcal{L}_{1}$ and $\mathcal{L}_{2}$ are atomistic lattices, then define $\mathcal{L}_{1} \otimes_{n} \mathcal{L}_{2}$ as $l_{1} \otimes_{n} l_{2}$, where $l_{i}=\left\{\Sigma\left[a_{i}\right] ; a_{i} \in \mathcal{L}_{i}\right\} \subseteq 2^{\Sigma_{i}}$ and $\Sigma\left[a_{i}\right]$ denotes the set of atoms under $a_{i}\left(\Sigma_{i}:=\Sigma\left[1_{i}\right]\right)$.

Proposition 3.10. Let $\mathcal{L}_{1}$ and $\mathcal{L}_{2}$ be lattices. Then $\mathcal{L}_{1} \square \mathcal{L}_{2}=L_{1} \oslash_{n} L_{2}$.

Proof. Direct from Definitions 3.7 and 3.9

Theorem 3.11. For atomistic lattices, $\mathcal{L}_{1} \oslash_{n} \mathcal{L}_{2} \cong \mathcal{L}_{1} \square \mathcal{L}_{2}$.

Proof. Define $f: \mathcal{L}_{1} \oslash_{n} \mathcal{L}_{2} \rightarrow \mathcal{L}_{1} \square \mathcal{L}_{2}$ as

$$
f\left(\Sigma\left[a_{1}\right] \times \Sigma_{2} \cup \Sigma_{1} \times \Sigma\left[a_{2}\right]\right)=a_{1} \square a_{2},
$$

and set $f\left(\bigcap x_{i}\right):=\bigcap f\left(x_{i}\right)$, where each $x_{i}$ has the form $\Sigma\left[a_{1}\right] \times \Sigma_{2} \cup \Sigma_{1} \times \Sigma\left[a_{2}\right]$. Obviously, $f$ is bijective and preserves meets, hence also joins.

3.3. The tensor products of Chu and Shmuely. We now compare $\oslash$ with three other tensor products of lattices appearing in the literature.

Definition 3.12 (P. H. Chu 2]). The category Chu(Set,2) has as objects triples $\mathrm{A}=(A, r, X)$ where $A$ and $X$ are sets and $r$ is a map from $A \times X$ to 2 (where $2=\{0,1\})$. Arrows are pairs of maps $(F, G): \mathrm{A} \rightarrow(B, s, Y)$ with $F: A \rightarrow B$ and $G: Y \rightarrow X$ such that $s(F(a), y)=r(a, G(y))$ for all $a \in A$ and $y \in Y$. There is a functor ${ }^{\perp}$ and a bifunctor $\underset{\mathrm{Chu}}{\otimes}$ defined on objects as $\mathrm{A}^{\perp}=(X, \check{r}, A)$ with $\check{r}(x, a)=r(a, x)$, and $\mathrm{A} \underset{\mathrm{Chu}}{\otimes} \mathrm{B}=\left(A \times B, t, \mathbf{C h u}\left(\mathrm{A}, \mathrm{B}^{\perp}\right)\right)$ with $t((a, b),(F, G))=$ $r(a, G(b))=\check{s}(F(a), b)$.

Remark 3.13. A simple closure space $\mathcal{L} \in \mathbf{C l}(\Sigma)$ can be identified with the Chu space $(\Sigma, r, \mathcal{L})$, which we also denote by $\mathcal{L}$, where $r(p, a)=1$ if and only if $p \in a$.

Proposition 3.14. Let $\mathcal{L}_{0}$ and $\mathcal{L}_{1}$ be simple closure spaces on $\Sigma_{0}$ and $\Sigma_{1}$ respectively. Then there is a one to one correspondence between invertible arrows in $\operatorname{Chu}\left(\mathcal{L}_{0}, \mathcal{L}_{1}\right)$ and isomorphisms between $\mathcal{L}_{0}$ and $\mathcal{L}_{1}$. 
Proof. Let $f: \mathcal{L}_{0} \rightarrow \mathcal{L}_{1}$ be an isomorphism. Then $\left(f, f^{-1}\right) \in \mathbf{C h u}\left(\mathcal{L}_{0}, \mathcal{L}_{1}\right)$. Let $(F, G)$ be an invertible arrow in $\mathbf{C h u}\left(\mathcal{L}_{0}, \mathcal{L}_{1}\right)$. Then $G: \mathcal{L}_{1} \rightarrow \mathcal{L}_{0}$ is bijective, and since $G=F^{-1}, G$ preserves arbitrary meets, hence also arbitrary joins.

Definition 3.15. Let $\mathcal{L}_{1}$ and $\mathcal{L}_{2}$ be posets. A Galois connection (more precisely an adjunction) between $\mathcal{L}_{1}$ and $\mathcal{L}_{2}$ is a pair $(f, g)$ of order-preserving maps with $f: \mathcal{L}_{1} \rightarrow \mathcal{L}_{2}$ and $g: \mathcal{L}_{2} \rightarrow \mathcal{L}_{1}$, such that for all $a \in \mathcal{L}_{1}$ and $b \in \mathcal{L}_{2}, f(a) \leq$ $b \Leftrightarrow a \leq g(b)$. Note that if $\mathcal{L}_{1}$ and $\mathcal{L}_{2}$ are complete lattices, then there is a one to one correspondence between maps from $\mathcal{L}_{1}$ to $\mathcal{L}_{2}$ preserving arbitrary joins and adjunctions between $\mathcal{L}_{1}$ and $\mathcal{L}_{2}$.

The following is standard; we include the easy proof for completeness.

Lemma 3.16. Let $\mathcal{L}_{0}$ and $\mathcal{L}_{1}$ be complete join-semilattices and $(f, g)$ a pair of maps with $f: \mathcal{L}_{0} \rightarrow \mathcal{L}_{1}$ and $g: \mathcal{L}_{1} \rightarrow \mathcal{L}_{0}$.

(1) If $(f, g)$ forms a Galois connection, then $f$ preserves arbitrary joins.

(2) Suppose that $\mathcal{L}_{0}$ is a simple closure space on $\Sigma_{0}$. For $b \in \mathcal{L}_{1}$, let $F^{-1}(b)$ denote the set $\left\{p \in \Sigma_{0} ; f(p) \leq b\right\}$. Then $f$ preserves arbitrary joins $\Leftrightarrow$ $f(a)=\bigvee\{f(p) ; p \in a\}$ for all $a \in \mathcal{L}_{0}$ and $F^{-1}(b) \in \mathcal{L}_{0}$ for all $b \in \mathcal{L}_{1}$.

Proof. (1) Let $\omega \subseteq \mathcal{L}_{0}$. Since $f$ preserves order, $y:=\bigvee\{f(x) ; x \in \omega\} \leq f(\bigvee \omega)$. On the other hand, for any $x \in \omega, f(x) \leq y$, hence $x \leq g(y)$. As a consequence, $\bigvee \omega \leq g(y)$, therefore $f(\bigvee \omega) \leq y$.

(2) $(\Rightarrow)$ If $p \in \bigvee F^{-1}(b)$, then $f(p) \leq \bigvee\left\{f(q) ; q \in F^{-1}(b)\right\} \leq b$, hence $p \in$ $F^{-1}(b)$. $(\Leftarrow)$ Obviously, $\left(f, F^{-1}\right)$ forms a Galois connection between $\mathcal{L}_{0}$ and $\mathcal{L}_{1}$.

Remark 3.17. Let $\mathcal{L}$ be a poset. Then $\mathcal{L}^{*}$ denotes the dual of $\mathcal{L}$ (defined by the converse order-relation) and $\leq_{*}$ the order relation in $\mathcal{L}^{*}$.

Theorem 3.18. Let $\mathcal{L}_{1}$ and $\mathcal{L}_{2}$ be simple closure spaces on $\Sigma_{1}$ and $\Sigma_{2}$ respectively. Then,

(1) $\mathcal{L}_{1} \otimes \mathcal{L}_{2} \cong \mathcal{L}_{1} \underset{\mathrm{Chu}}{\otimes} \mathcal{L}_{2}$

(2) $\left(\mathcal{L}_{1} \otimes \mathcal{L}_{2}\right)^{*}$ is isomorphic to the set of join-preserving maps $\mathcal{L}_{1} \rightarrow \mathcal{L}_{2}^{*}$, ordered point-wise (i.e., $f \leq g$ if and only if $f\left(p_{1}\right) \leq_{*} g\left(p_{1}\right)$ for all $p_{1} \in \Sigma_{1}$ ).

Proof. (1) We denote $\Sigma_{1} \times \Sigma_{2}$ by $\boldsymbol{\Sigma}$. Let $(F, G) \in \mathbf{C h u}\left(\mathcal{L}_{1}, \mathcal{L}_{2}^{\perp}\right)$. Define

$$
R_{(F, G)}:=\bigcup_{p_{1} \in \Sigma_{1}} p_{1} \times F\left(p_{1}\right)
$$

By definition, $R_{(F, G)_{2}}[p] \in \mathcal{L}_{2}$ for all $p \in \boldsymbol{\Sigma}$, and

$$
q_{1} \in R_{(F, G)}[p] \Leftrightarrow\left(q_{1}, p_{2}\right) \in R_{(F, G)} \Leftrightarrow p_{2} \in F\left(q_{1}\right) \Leftrightarrow q_{1} \in G\left(p_{2}\right),
$$

thus $R_{(F, G)_{1}}[p] \in \mathcal{L}_{1}$ for all $p \in \Sigma$, hence $R_{(F, G)} \in \mathcal{L}_{1} \otimes \mathcal{L}_{2}$.

Let $R \in \mathcal{L}_{1} \otimes \mathcal{L}_{2}$. Define $F_{R}: \Sigma_{1} \rightarrow \mathcal{L}_{2}$ and $G_{R}: \Sigma_{2} \rightarrow \mathcal{L}_{1}$ as $F_{R}\left(p_{1}\right):=R_{2}\left[\left(p_{1}, \cdot\right)\right]$ and $G_{R}\left(p_{2}\right):=R_{1}\left[\left(\cdot, p_{2}\right)\right]$. Then

$$
p_{2} \in F_{R}\left(p_{1}\right) \Leftrightarrow\left(p_{1}, p_{2}\right) \in R \Leftrightarrow p_{1} \in G_{R}\left(p_{2}\right),
$$

so that $\left(F_{R}, G_{R}\right) \in \mathbf{C h u}\left(\mathcal{L}_{1}, \mathcal{L}_{2}^{\perp}\right)$. Note that $(F, G) \mapsto R_{(F, G)}$ is isotone in both directions.

Obviously,

$$
F_{R_{(F, G)}}=F, G_{R_{(F, F)}}=G \text {, and } R_{\left(F_{R}, G_{R}\right)}=R \text {. }
$$


As a consequence, there is an invertible arrow in $\operatorname{Chu}\left(\mathcal{L}_{1} \otimes \mathcal{L}_{2}, \mathcal{L}_{1} \otimes \mathcal{L}_{2}\right)$.

(2) Let $(F, G) \in \operatorname{Chu}\left(\mathcal{L}_{1}, \mathcal{L}_{2}^{\perp}\right)$. Define $f: \mathcal{L}_{1} \rightarrow \mathcal{L}_{2}^{*}$ and $g: \mathcal{L}_{2}^{*} \rightarrow \mathcal{L}_{1}$ as $f(a):=\bigcap F(a)$ and $g(b)=\bigcap G(b)$. Then, obviously $(f, g)$ forms a Galois connection between $\mathcal{L}_{1}$ and $\mathcal{L}_{2}^{*}$.

Let $f: \mathcal{L}_{1} \rightarrow \mathcal{L}_{2}^{*}$ be $\bigvee$-preserving. Define $g: \mathcal{L}_{2} \rightarrow \mathcal{L}_{1}$ as $g(b):=\bigvee\{a \in$ $\left.\mathcal{L}_{1} ; f(a) \leq_{*} b\right\}$. Then, obviously $(f, g) \in \mathbf{C h u}\left(\mathcal{L}_{1}, \mathcal{L}_{2}^{\perp}\right)$.

Remark 3.19. Note that $\emptyset$ is the $\otimes$-tensor product of Golfin [6]. As a corollary of Theorem 3.18 part $(2), \mathcal{L}_{1} \otimes \mathcal{L}_{2} \cong \mathcal{L}_{1} \otimes \mathcal{L}_{2}$ the tensor product of Shmuely [12].

3.4. The semilattice tensor product of Fraser. We now consider tensor products as solutions to universal mapping problems.

Definition 3.20. Let $\mathbf{C}$ be a concrete category over Set. Let $\left\{A_{\alpha}\right\}_{\alpha \in \Omega}$ and $C$ be objects of $\mathbf{C}$. A multimorphism of $\mathbf{C}$ is an arrow $f \in \operatorname{Set}\left(\prod_{\alpha} \mathrm{A}_{\alpha}, \mathrm{C}\right)$ such that for all $a \in \prod_{\alpha} \mathrm{A}_{\alpha}, f(a[-, \alpha]) \in \mathbf{C}\left(\mathrm{A}_{\alpha}, \mathrm{C}\right)$ (see Definition [2.3). If $|\Omega|=2$, then $f$ is called a bimorphism.

Definition 3.21 (G. Seal, [1]). Let $\mathbf{C}$ be a concrete category over Set. A tensor product in $\mathbf{C}$ is a bifunctor $-\otimes-: \mathbf{C} \times \mathbf{C} \rightarrow \mathbf{C}$ such that for all objects $A$, B of $\mathbf{C}$, there is a a bimorphism $f: A \times B \rightarrow A \otimes B$, and for all object $C$ of $\mathbf{C}$, and all bimorphism $g: \mathrm{A} \times \mathrm{B} \rightarrow \mathrm{C}$, there is a unique arrow $h \in \mathbf{C}(\mathrm{A} \otimes \mathrm{B}, \mathrm{C})$ which makes the diagram

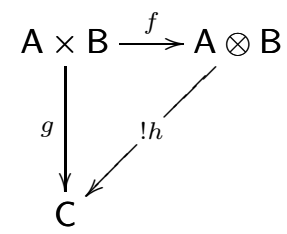

commute.

Remark 3.22. By definition, the tensor product is unique up to isomorphisms. Consider now the category $\mathbf{C}$ of join-semilattices with maps preserving finite joins. Let $\mathcal{L}_{1}, \mathcal{L}_{2}$ and $\mathcal{L}$ be join-semilattices. If $\mathcal{L}$ is a tensor product of $\mathcal{L}_{1}$ and $\mathcal{L}_{2}$ in $\mathbf{C}$, then $f\left(\mathcal{L}_{1} \times \mathcal{L}_{2}\right)$ generates $\mathcal{L}$. Indeed, let $\mathcal{L}_{0}$ be a (join-semilattice) tensor product of $\mathcal{L}_{1}$ and $\mathcal{L}_{2}$ as defined by Fraser in [5] (write $g$ the bimorphism from $\mathcal{L}_{1} \times \mathcal{L}_{2}$ to $\left.\mathcal{L}_{0}\right)$. Then by definition $\mathcal{L}_{0}$ is generated by $g\left(\mathcal{L}_{1} \times \mathcal{L}_{2}\right)$, and obviously $\mathcal{L}_{0}$ is a tensor product of $\mathcal{L}_{1}$ and $\mathcal{L}_{2}$ in $\mathbf{C}$. Therefore there is an isomorphism $h: \mathcal{L}_{0} \rightarrow \mathcal{L}$ such that $f=h \circ g$. Hence, for every $x \in \mathcal{L}$ we have $x=h\left(h^{-1}(x)\right)=h\left(\bigvee g\left(a_{i}, b_{i}\right)\right)=$ $\bigvee h\left(g\left(a_{i}, b_{i}\right)\right)=\bigvee f\left(a_{i}, b_{i}\right)$ where $a_{i} \in \mathcal{L}_{1}$ and $b_{i} \in \mathcal{L}_{2}$

As a consequence, for the category of join-semilattices with maps preserving finite joins, Definition 3.21 is equivalent to the definition of Fraser.

Theorem 3.23. $\oslash$ is a tensor product in $\mathbf{C l}$.

Proof. Let $\mathcal{L}_{1}, \mathcal{L}_{2} \in \mathbf{C l}$. By Theorem 2.12, $\mathcal{L}_{1} \otimes \mathcal{L}_{2} \in \mathbf{C l}$, and by Lemma 2.7], the map $f: \mathcal{L}_{1} \times \mathcal{L}_{2} \rightarrow \mathcal{L}_{1} \otimes \mathcal{L}_{2}$ sending $a=\left(a_{1}, a_{2}\right)$ to $\bar{a}=a_{1} \times a_{2}$ is a bimorphism.

Let $\mathcal{L} \in \mathbf{C l}$ (or a complete join-semilattice), and let $g: \mathcal{L}_{1} \times \mathcal{L}_{2} \rightarrow \mathcal{L}$ be a bimorphism. Define $h: \mathcal{L}_{1} \oslash \mathcal{L}_{2} \rightarrow \mathcal{L}$ by $h(R)=\bigvee\left\{g\left(p_{1}, p_{2}\right) ;\left(p_{1}, p_{2}\right) \in R\right\}$. Furthermore, for $p_{1} \in \Sigma_{1}$ and $p_{2} \in \Sigma_{2}$, define $g_{p_{1}}: \mathcal{L}_{2} \rightarrow \mathcal{L}$ and $g_{p_{2}}: \mathcal{L}_{1} \rightarrow \mathcal{L}$ as $g_{p_{1}}(y):=g\left(p_{1}, y\right)$ and $g_{p_{2}}(x):=g\left(x, p_{2}\right)$ respectively. Let $b \in \mathcal{L}$. Write, as in 
Lemma 3.16 $H^{-1}(b)=\left\{p \in \Sigma_{1} \times \Sigma_{2} ; h(p) \leq b\right\}, G_{p_{1}}^{-1}(b)=\left\{s \in \Sigma_{2} ; g_{p_{1}}(s) \leq b\right\}$, and $G_{p_{2}}^{-1}(b)=\left\{r \in \Sigma_{1} ; g_{p_{2}}(r) \leq b\right\}$. Then,

$$
H^{-1}(b)=\bigcup_{p_{1} \in \Sigma_{1}} p_{1} \times G_{p_{1}}^{-1}(b)=\bigcup_{p_{2} \in \Sigma_{2}} G_{p_{2}}^{-1}(b) \times p_{2} .
$$

Since $g$ is a bimorphism, it follows from Lemma 3.16] and Definition 2.9 that $H^{-1}(b) \in \mathcal{L}_{1} \otimes \mathcal{L}_{2}$. Therefore, by Lemma 3.16, $h \in \operatorname{Cl}\left(\mathcal{L}_{1} \otimes \mathcal{L}_{2}, \mathcal{L}\right)$. Finally, if $h^{\prime} \in \mathbf{C l}\left(\mathcal{L}_{1} \otimes \mathcal{L}_{2}, \mathcal{L}\right)$ and $h^{\prime} \circ f=g$, then $h^{\prime}$ equals $h$ on atoms, therefore $h^{\prime}=h$.

Let $L_{1}, L_{2} \in \mathbf{C l}, f_{1} \in \mathbf{C l}\left(\mathcal{L}_{1}, L_{1}\right)$, and $f_{2} \in \mathbf{C l}\left(\mathcal{L}_{2}, L_{2}\right)$. Then $g=f \circ\left(f_{1} \times f_{2}\right)$ : $\mathcal{L}_{1} \times \mathcal{L}_{2} \rightarrow L_{1} \otimes L_{2}$ is a bimorphism. We define $f_{1} \otimes f_{2} \in \mathbf{C l}\left(\mathcal{L}_{1} \otimes \mathcal{L}_{2}, L_{1} \otimes L_{2}\right)$ to be the arrow $h$ constructed above.

Remark 3.24. Let $\mathbf{C}_{\text {com }}$ denote the category of complete join-semilattices with maps preserving arbitrary joins. Let $\mathcal{L}_{1}$ and $\mathcal{L}_{2} \in$ Cl. Then $\mathcal{L}_{1}, \mathcal{L}_{2}, \mathcal{L}_{1} \otimes \mathcal{L}_{2} \in$ $\mathbf{C}_{\text {com }}$. Moreover, we have proved that $\mathcal{L}_{1} \otimes \mathcal{L}_{2}$ is the tensor product of $\mathcal{L}_{1}$ and $\mathcal{L}_{2}$ in $\mathbf{C}_{\text {com }}$.

\section{Equivalent DEFinition}

In this section we give an equivalent definition of $\mathrm{S}\left(\mathcal{L}_{\alpha}, \alpha \in \Omega\right)$ and $\mathcal{S}_{T}\left(\mathcal{L}_{\alpha}, \alpha \in \Omega\right)$ in terms of a universal property with respect to a given class of bimorphisms of $\mathbf{C l}$.

Lemma 4.1. Let $\mathcal{L}$ be a simple closure space on $\Sigma$. For all $a \in \mathcal{L}$, there is a unique mapping $h_{a}: \mathcal{L} \rightarrow 2$ preserving arbitrary joins such that for all $p \in \Sigma, h_{a}(p)=0$ if and only if $p \in a$.

Proof. Let $b \in \mathcal{L}$. Define $h_{a}$ by $h_{a}(b)=\bigvee\left\{h_{a}(p) ; p \in b\right\}$ (hence $h_{a}(0)=0$ ) with $h_{a}(p)=0$ if $p \in a$ and $h_{a}(p)=1$ if $p \notin a$. Then, by Lemma 3.16, $h$ preserves arbitrary joins.

Lemma 4.2. Let $\mathcal{L}$ and $\mathcal{L}_{\alpha}(\alpha \in \Omega)$ be simple closure spaces. Then

(1) If $f: \prod_{\alpha} \mathcal{L}_{\alpha} \rightarrow \mathcal{L}$ a multimorphism of $\mathbf{C l}$ and $v \in \prod_{\alpha} \operatorname{Aut}\left(\mathcal{L}_{\alpha}\right)$, then $f \circ v$ is again a multimorphism.

(2) For $g \in \prod_{\alpha} \mathbf{C l}\left(\mathcal{L}_{\alpha}, 2\right)$, the mapping $\bigcap_{\alpha} g_{\alpha}: \prod_{\alpha} \mathcal{L}_{\alpha} \rightarrow 2$, defined as

$$
\bigcap_{\alpha} g_{\alpha}(a):=\bigcap\left\{g_{\alpha}\left(a_{\alpha}\right) ; \alpha \in \Omega\right\},
$$

is a multimorphism.

Proof. (1) Let $w=f \circ v, a \in \prod_{\alpha} \mathcal{L}_{\alpha}, \beta \in \Omega$, and $\omega \subseteq \mathcal{L}_{\beta}$. Then

$$
w(a[\bigvee \omega, \beta])=f\left(v(a)\left[\bigvee\left\{v_{\beta}(x) ; x \in \omega\right\}, \beta\right]\right)=\bigvee_{x \in \omega} w(a[x, \beta]),
$$

since $f$ is a multimorphism.

(2) Let $g=\bigcap_{\alpha} g_{\alpha}, a \in \prod_{\alpha} \mathcal{L}_{\alpha}, \beta \in \Omega$, and $b \in \mathcal{L}$. Write

$$
h(-):=g(a[-, \beta]): \mathcal{L}_{\beta} \rightarrow 2 .
$$

Then $h(b)=g_{\beta}(b) \bigcap_{\alpha \neq \beta} g_{\alpha}\left(a_{\alpha}\right)$. Hence $h(b)=0$ if $g_{\alpha}\left(a_{\alpha}\right)=0$ for $\alpha \neq \beta$ and $h(b)=g_{\beta}(b)$ otherwise. As a consequence, $h$ preserves arbitrary joins, hence $g$ is a multimorphism of $\mathbf{C l}$. 
Definition 4.3. Let $\mathcal{L}$ and $\mathcal{L}_{\alpha}(\alpha \in \Omega)$ be simple closure spaces, $f: \prod_{\alpha} \mathcal{L}_{\alpha} \rightarrow \mathcal{L}$ a multimorphism of $\mathbf{C l}$ and $T_{\alpha} \subseteq \operatorname{Aut}\left(\mathcal{L}_{\alpha}\right)$. Put $T:=\prod_{\alpha} T_{\alpha}$. Then, we define

$$
\begin{aligned}
V_{T}(f) & :=\left\{f \circ v: \prod_{\alpha} \mathcal{L}_{\alpha} \rightarrow \mathcal{L} ; v \in T\right\}, \\
\Gamma_{\cap} & :=\left\{\bigcap_{\alpha} g_{\alpha}: \prod_{\alpha} \mathcal{L}_{\alpha} \rightarrow 2 ; g \in \prod_{\alpha} \mathbf{C l}\left(\mathcal{L}_{\alpha}, 2\right)\right\} .
\end{aligned}
$$

Definition 4.4. Let $\mathcal{L}$ be a simple closure space and $A \subseteq \mathcal{L}$. We say that $A$ generates $\mathcal{L}$ if each element of $\mathcal{L}$ is the join of elements in $A$.

Theorem 4.5. Let $\left\{\mathcal{L}_{\alpha}\right\}_{\alpha \in \Omega}$ be a family of simple closure spaces on $\Sigma_{\alpha}, \mathcal{L}$ a simple closure space on $\boldsymbol{\Sigma}$, and $T=\prod_{\alpha} T_{\alpha}$ with $T_{\alpha} \subseteq \operatorname{Aut}\left(\mathcal{L}_{\alpha}\right)$.

(1) If $\mathcal{L} \in \mathcal{S}_{T}\left(\mathcal{L}_{\alpha}, \alpha \in \Omega\right)$, then there is a multimorphism $f: \prod_{\alpha} \mathcal{L}_{\alpha} \rightarrow \mathcal{L}$ of $\mathbf{C l}$ such that $f\left(\prod_{\alpha} \mathcal{L}_{\alpha}\right)$ generates $\mathcal{L}$, and for all $g \in \Gamma_{\cap}$ and $w \in V_{T}(f)$, there is a unique $h \in \mathbf{C l}(\mathcal{L}, 2)$ and a unique $u \in \mathbf{C l}(\mathcal{L}, \mathcal{L})$ such that the following diagrams commute.
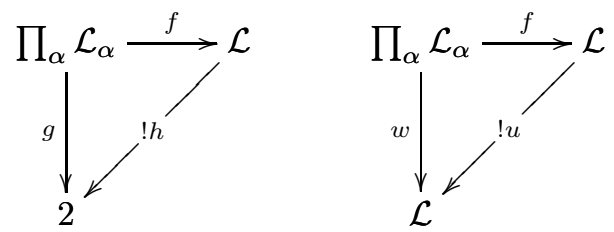

(2) Conversely, if there is a multimorphism of $\mathbf{C l} f: \prod_{\alpha} \mathcal{L}_{\alpha} \rightarrow \mathcal{L}$ satisfying all conditions of part (1), then there is some $\mathcal{L}_{0} \in \mathcal{S}_{T}\left(\mathcal{L}_{\alpha}, \alpha \in \Omega\right)$ such that $\mathcal{L} \cong \mathcal{L}_{0}$

Proof. (1) By Lemma 2.7], the map $f: \prod_{\alpha} \mathcal{L}_{\alpha} \rightarrow \mathcal{L}$ sending $a$ to $\bar{a}$, is a multimorphism of $\mathbf{C l}$, and obviously, $f\left(\prod_{\alpha} \mathcal{L}_{\alpha}\right)$ generates $\mathcal{L}$. Moreover, by Axiom $\mathrm{P} 4$, for all $w \in V_{T}(f)$ there is a unique $u$ such that the second diagram commutes.

Let $g=\bigcap_{\alpha} g_{\alpha} \in \Gamma_{\cap}$. Write $G_{\alpha}$ for the restriction to atoms of $g_{\alpha}$. Let $a_{\alpha}:=$ $\bigvee G_{\alpha}^{-1}(0)$. Note that by Lemma 3.16, $a_{\alpha}=G_{\alpha}^{-1}(0)$. Recall that by hypothesis, $\mathcal{L} \in \mathbf{C l}(\boldsymbol{\Sigma})$ with $\boldsymbol{\Sigma}=\prod_{\alpha} \Sigma_{\alpha}$. Define $x:=\bigcup_{\alpha} \pi_{\alpha}^{-1}\left(a_{\alpha}\right)$. By Axiom P2 $x \in \mathcal{L}$, and by Lemma 4.1 there is $h_{x} \in \mathbf{C l}(\mathcal{L}, 2)$ such that for all $p \in \boldsymbol{\Sigma}, h_{x}(p)=0$ if and only if $p \in x$. Hence, $h_{x} \circ f=g$. Let $h \in \mathbf{C l}(\mathcal{L}, 2)$ such that $h \circ f=g$. Then on atoms $h$ equals $h_{x}$, therefore $h=h_{x}$.

(2) Let $\boldsymbol{\Sigma}_{\mathbf{0}}=\prod_{\alpha} \Sigma_{\alpha}$. We denote by $F$ the mapping from $\boldsymbol{\Sigma}_{\mathbf{0}}$ to $\mathcal{L}$ induced by the multimorphism $f$.

(2.1) For $a, b \in \prod_{\alpha} \mathcal{L}_{\alpha} \backslash\left\{0_{\alpha}\right\}$, we write $a \leq b$ if and only if $a_{\beta} \leq b_{\beta}$, for all $\beta \in \Omega$ (this is the standard product ordering on $\prod_{\alpha} \mathcal{L}_{\alpha}$ ). Claim: $f(a) \subseteq f(b) \Rightarrow a \leq b$. As a corollary, $f$ is injective. [Proof: Suppose that $f(a) \subseteq f(b)$ and that $a_{\beta} \not \leq b_{\beta}$ for some $\beta \in \Omega$. By Lemma 4.1 there is $h_{b_{\beta}} \in \mathbf{C l}\left(\mathcal{L}_{\beta}, 2\right)$ such that for all $p \in \Sigma_{\beta}$, $h_{b_{\beta}}(p)=0$ if and only if $p \in b_{\beta}$. Let $g_{\beta}:=h_{b_{\beta}}, g_{\alpha}:=h_{0_{\alpha}}$ for all $\alpha \neq \beta$, and $g:=\bigcap_{\alpha} g_{\alpha}$. By definition, $g \in \Gamma_{\cap}$, hence there is $h \in \mathbf{C l}(\mathcal{L}, 2)$ such that $h \circ f=g$. As a consequence,

$$
1=g_{\beta}\left(a_{\beta}\right)=g(a)=h(f(a)) \subseteq h(f(b))=g(b)=g_{\beta}\left(b_{\beta}\right)=0,
$$

a contradiction. This proves the claim.]

As a consequence, since $f\left(\prod_{\alpha} \mathcal{L}_{\alpha}\right)$ generates $\mathcal{L}$, for all $p \in \boldsymbol{\Sigma}_{\mathbf{0}}, F(p)$ is an atom of $\mathcal{L}$, and the mapping from $\boldsymbol{\Sigma}_{\mathbf{0}}$ to $\boldsymbol{\Sigma}$ induced by $F$ (which we also denote by $F$ ) is 
bijective. Moreover, for all $a \in \prod_{\alpha} \mathcal{L}_{\alpha}$,

$$
f(a)=\bigvee\{F(p) ; p \in \bar{a}\}
$$

Therefore, for all $a, b \in \prod_{\alpha} \mathcal{L}_{\alpha}$, we have: $a \leq b \Rightarrow f(a) \subseteq f(b)$ (note that if $\Omega$ is finite, then this implication follows directly from the fact that $f$ is a multimorphism).

(2.2) Let $\mathcal{L}_{0} \subseteq 2^{\Sigma_{0}}$ defined as $\mathcal{L}_{0}:=\left\{F^{-1}(c) ; c \in \mathcal{L}\right\}$. Then by what precedes, $\mathcal{L}_{0} \in \mathbf{C l}\left(\boldsymbol{\Sigma}_{\mathbf{0}}\right)$ and the map $F^{-1}: \mathcal{L} \rightarrow \mathcal{L}_{0}$ is bijective and preserves arbitrary meets, hence also arbitrary joins. It remains to prove that $\mathcal{L}_{0} \in \mathcal{S}_{T}\left(\mathcal{L}_{\alpha}, \alpha \in \Omega\right)$, hence to check that Axioms P2, P3 and P4 hold in $\mathcal{L}_{0}$. Below, if $g \in \Gamma_{\cap}$, then $G$ denotes the map from $\boldsymbol{\Sigma}_{\mathbf{0}}$ to 2 induced by $g$.

(P2) Let $a \in \prod_{\alpha} \mathcal{L}_{\alpha}, x:=F\left(\bigcup_{\alpha} \pi_{\alpha}^{-1}\left(a_{\alpha}\right)\right)$, and $p \in \boldsymbol{\Sigma}_{\mathbf{0}}$ such that $p_{\alpha} \notin a_{\alpha}$ for all $\alpha \in \Omega$. Suppose that $F(p) \in \bigvee x$. From Lemma 4.1, for all $\beta \in \Omega$, there is $h_{a_{\beta}} \in \mathbf{C l}\left(\mathcal{L}_{\beta}, 2\right)$ such that for all $p \in \Sigma_{\beta}, h_{a_{\beta}}(p)=0$ if and only if $p \in a_{\beta}$. Let $g=\bigcap_{\alpha} h_{a_{\alpha}}$. By definition, $g \in \Gamma_{\cap}$, hence there is $h \in \mathbf{C l}(\mathcal{L}, 2)$ such that $h \circ f=g$; whence

$$
\begin{aligned}
1=G(p)=h(F(p)) \subseteq h(\bigvee x) & =\bigvee\left\{h(F(p)) ; p \in \bigcup_{\alpha} \pi_{\alpha}^{-1}\left(a_{\alpha}\right)\right\} \\
& =\bigvee\left\{G(p) ; p \in \bigcup_{\alpha} \pi_{\alpha}^{-1}\left(a_{\alpha}\right)\right\}=0,
\end{aligned}
$$

a contradiction. As a consequence, $F^{-1}\left(\bigvee F\left(\bigcup_{\alpha} \pi_{\alpha}^{-1}\left(a_{\alpha}\right)\right)\right)=\bigcup_{\alpha} \pi_{\alpha}^{-1}\left(a_{\alpha}\right)$, hence $\bigcup_{\alpha} \pi_{\alpha}^{-1}\left(a_{\alpha}\right) \in \mathcal{L}_{0}$

(P3) Let $\beta \in \Omega, A \subseteq \Sigma_{\beta}$, and $p \in \mathbf{\Sigma}_{\mathbf{0}}$, such that $p[A, \beta] \in \mathcal{L}_{0}$, i.e., there is $c \in \mathcal{L}$ such that $p[A, \beta]=F^{-1}(c)$. Let $q \in \bigvee A$ and $\hat{p} \in \prod_{\alpha} \mathcal{L}_{\alpha}$ such that $\bar{p}=\{p\}$. Then $p[q, \beta] \in p[\bigvee A, \beta]$, hence, since $f$ is multimorphism, we find that

$$
F(p[q, \beta]) \in F(p[\bigvee A, \beta]) \subseteq \bigvee F(p[\bigvee A, \beta])=f(\hat{p}[\bigvee A, \beta])=\bigvee_{q \in A} F(p[q, \beta]) \subseteq c
$$

As a consequence, $q \in A$, therefore $A \in \mathcal{L}_{\beta}$.

$(\mathrm{P} 4)$ Let $v \in T$ and $u \in \mathbf{C l}(\mathcal{L}, \mathcal{L})$ such that $u \circ f=f \circ v$. Define $u_{0}:=F^{-1} \circ u \circ F$. Then $u_{0} \in \operatorname{Aut}\left(\mathcal{L}_{0}\right)$ and $u_{0}(p)_{\alpha}=v_{\alpha}\left(p_{\alpha}\right)$ for all $p \in \Sigma_{0}$ and all $\alpha \in \Omega$.

\section{Central elements}

Let $\left\{\mathcal{L}_{\alpha}\right\}_{\alpha \in \Omega}$ be a family of simple closure spaces and $\beta \in \Omega$. In this section we prove that if $z$ is a central element of $\mathcal{L}_{\beta}$, then $\pi_{\beta}^{-1}(z)$ is a central element of $\otimes_{\alpha} \mathcal{L}_{\alpha}$ and of $\otimes_{\alpha} \mathcal{L}_{\alpha}$. As a corollary, if $\otimes_{\alpha} \mathcal{L}_{\alpha}$ or if $\otimes_{\alpha} \mathcal{L}_{\alpha}$ is irreducible, then all $\mathcal{L}_{\alpha}$ 's are irreducible. We give some sufficient conditions under which the converse result holds in $\otimes_{\alpha} \mathcal{L}_{\alpha}$.

Definition 5.1. Let $a$ and $b$ be elements of a lattice. Then $(a, b)$ is said to be a modular pair (in symbols $(a, b) M)$ if $(c \vee a) \wedge b=c \vee(a \wedge b)$ for all $c \leq b$.

Lemma 5.2. Let $\mathcal{L}$ be a simple closure space on $\Sigma$ and $z \in \mathcal{L}$. Then $z$ is a central element of $\mathcal{L}$ if and only if $z^{c}:=\Sigma \backslash z \in \mathcal{L}$ and $\left(z, z^{c}\right) M$ and $\left(z^{c}, z\right) M$.

Proof. Direct from Theorem $4.13(\varepsilon)$ in [10].

Corollary 5.3. Let $\mathcal{L}$ be a simple closure space on $\Sigma$, $z$ a central element, $a \subseteq z$, and $b \subseteq z^{c}$. Then $a \vee b=a \cup b$. 
Proof. From $\left(z^{c}, z\right) M$ follows that $(a \vee b) \cap z \subseteq\left(a \vee z^{c}\right) \cap z=a$, and from $\left(z, z^{c}\right) M$ follows that $(a \vee b) \cap z^{c} \subseteq(b \vee z) \cap z^{c}=b$.

Theorem 5.4. Let $\left\{\mathcal{L}_{\alpha}\right\}_{\alpha \in \Omega}$ be a family of simple closure spaces on $\Sigma_{\alpha}$ and $\beta \in \Omega$. If $z$ is a central element of $\mathcal{L}_{\beta}$, then $\pi_{\beta}^{-1}(z)$ is a central element of $\otimes_{\alpha} \mathcal{L}_{\alpha}$.

Proof. We denote $\pi_{\beta}^{-1}(z)$ by $Z$. Hence $Z^{c}=\pi_{\beta}^{-1}\left(z^{c}\right)$. From Lemma 5.2, $z^{c}:=$ $\Sigma_{\beta} \backslash z \in \mathcal{L}_{\beta}$. Therefore, by Axiom P2, $Z^{c} \in \bigotimes_{\alpha} \mathcal{L}_{\alpha}$.

We now prove that $\left(Z, Z^{c}\right) M$. The proof for $\left(Z^{c}, Z\right) M$ is similar. Let $R \in \bigotimes_{\alpha} \mathcal{L}_{\alpha}$ with $R \subseteq Z^{c}$. Let $\boldsymbol{\Sigma}=\prod_{\alpha} \Sigma_{\alpha}, p \in \boldsymbol{\Sigma}, X=R \cup Z$, and $\alpha \in \Omega$. If $\alpha \neq \beta$, then $X_{\alpha}[p]=\Sigma_{\alpha}$ if $p_{\beta} \in z$ and $X_{\alpha}[p]=R_{\alpha}[p]$ otherwise. On the other hand, $X_{\beta}[p]=$ $z \cup R_{\beta}[p]$, hence $X_{\beta}[p] \in \mathcal{L}_{\beta}$ by Corollary [5.3. As a consequence, $R \vee Z=R \cup Z$, therefore $(R \vee Z) \cap Z^{c}=(R \cup Z) \cap Z^{c}=R$.

Theorem 5.5 (9], Theorem 1). Let $\left\{\mathcal{L}_{\alpha}\right\}_{\alpha \in \Omega}$ be a family of orthocomplemented simple closure spaces on $\Sigma_{\alpha}$. Suppose that one of the following assumptions holds.

(1) $\Omega$ is finite.

(2) For all $\alpha \in \Omega, \mathcal{L}_{\alpha}$ has the covering property, and for all $p \neq q \in \Sigma_{\alpha}$ having the same central cover, $p \vee q$ contains an infinite number of atoms.

Then, $\otimes_{\alpha} \mathcal{L}_{\alpha}$ is irreducible if and if all $\mathcal{L}_{\alpha}$ 's are irreducible.

\section{Automorphisms}

In this section we prove the following result. Let $\mathcal{L}_{1}, \cdots, \mathcal{L}_{n}$ be simple closure spaces different from $2, \mathcal{L} \in \mathrm{S}\left(\mathcal{L}_{1}, \cdots, \mathcal{L}_{n}\right)$ and $u \in \mathbf{C l}(\mathcal{L}, \mathcal{L})$ sending atoms to atoms. If $u$ is large (see Definition 6.3 below), then there is a permutation $f$ of $\{1, \cdots, n\}$ and arrows $v_{i} \in \mathbf{C l}\left(\mathcal{L}_{i}, \mathcal{L}_{f(i)}\right)$ sending atoms to atoms such that for any atom $p$ of $\mathcal{L}, u(p)_{f(i)}=v_{i}\left(p_{i}\right)$. We need some hypotheses on each $\mathcal{L}_{i}$ which are true for instance if each $\mathcal{L}_{i}$ is irreducible orthocomplemented with the covering property or an irreducible DAC-lattice. Note that our hypotheses imply irreducibility. Note also that if $u$ is an automorphism, then $u$ is large.

Definition 6.1. Let $\mathcal{L}$ be a simple closure space on $\Sigma$. We say that $\mathcal{L}$ is weakly connected if $\mathcal{L} \neq 2$ and if there is a connected covering of $\Sigma$, that is a family of subsets $\left\{A^{\gamma} \subseteq \Sigma ; \gamma \in \sigma\right\}$ such that

(1) $\Sigma=\bigcup\left\{A^{\gamma} ; \gamma \in \sigma\right\}$ and $\left|A^{\gamma}\right| \geq 2$ for all $\gamma \in \sigma$,

(2) for all $\gamma \in \sigma$ and all $p \neq q \in A^{\gamma}, p \vee q$ contains a third atom,

(3) for all $p, q \in \Sigma$, there is a finite subset $\left\{\gamma_{1}, \cdots, \gamma_{n}\right\} \subseteq \sigma$ such that $p \in A^{\gamma_{1}}$, $q \in A^{\gamma_{n}}$, and such that $\left|A^{\gamma_{i}} \cap A^{\gamma_{i+1}}\right| \geq 2$ for all $1 \leq i \leq n-1$.

We say that $\mathcal{L}$ is connected if $\mathcal{L} \neq 2$ and for all $p, q \in \Sigma, p \vee q$ contains a third atom, say $r$, such that $p \in q \vee r$ and $q \in p \vee r$.

Remark 6.2. Note that in part (2) of Definition 6.1, it is not required that the third atom under $p \vee q$ is in $A^{\gamma}$. Note also that by Corollary 5.3, weakly connected implies irreducible. Finally, let $\mathcal{L}$ be a simple closure space. Then, if $\mathcal{L} \neq 2$ and $\mathcal{L}$ is irreducible orthocomplemented with the covering property or an irreducible DAC-lattice, then $\mathcal{L}$ is connected.

Definition 6.3. Let $\left\{\mathcal{L}_{\alpha}\right\}_{\alpha \in \Omega}$ be a family of simple closure spaces on $\Sigma_{\alpha}$ different from 2. Let $\mathcal{L} \in \mathrm{S}\left(\mathcal{L}_{\alpha}, \alpha \in \Omega\right), \boldsymbol{\Sigma}=\prod_{\alpha} \Sigma_{\alpha}$, and let $u \in \mathbf{C l}(\mathcal{L}, \mathcal{L})$. We say that $u$ is large if for all $\beta \in \Omega$ and $p \in \boldsymbol{\Sigma}, u\left(p\left[\Sigma_{\beta}\right]\right)$ is not an atom of $\mathcal{L}$, and $u(1) \nsubseteq \pi_{\beta}^{-1}\left(p_{\beta}\right)$. 
Lemma 6.4. Let $\left\{\mathcal{L}_{\alpha}\right\}_{\alpha \in \Omega}$ be a family of simple closure spaces on $\Sigma_{\alpha}, \boldsymbol{\Sigma}=\prod_{\alpha} \Sigma_{\alpha}$, and let $\mathcal{L}$ be a simple closure space on $\boldsymbol{\Sigma}$. Suppose that Axiom P2 holds in $\mathcal{L}$. Let $p, q \in \mathbf{\Sigma}$.

(1) If $p_{\beta} \neq q_{\beta}$ for at least two $\beta \in \Omega$, then $p \vee q=p \cup q$.

(2) For all $\beta \neq \gamma \in \Omega$ and for all $b \in \mathcal{L}_{\beta}$ and $c \in \mathcal{L}_{\gamma}$ such that $p_{\beta} \in b$ and $p_{\gamma} \in c, p[b, \beta] \vee p[c, \gamma]=p[b, \beta] \cup p[c, \gamma]$.

Proof. (1) Let $r \in p \vee q$, and suppose that $p_{\beta} \neq q_{\beta}$ and $p_{\gamma} \neq q_{\gamma}$ for some $\beta \neq \gamma \in \Omega$. We must show that $r \in p \cup q$, i.e., that $r=p$ or $r=q$. Now

$$
\begin{aligned}
p, q \in\left(\pi_{\beta}^{-1}\left(p_{\beta}\right) \cup \pi_{\gamma}^{-1}\left(q_{\gamma}\right)\right) & \cap\left(\pi_{\beta}^{-1}\left(q_{\beta}\right) \cup \pi_{\gamma}^{-1}\left(p_{\gamma}\right)\right) \\
& =\left(\pi_{\beta}^{-1}\left(p_{\beta}\right) \cap \pi_{\gamma}^{-1}\left(p_{\gamma}\right)\right) \cup\left(\pi_{\beta}^{-1}\left(q_{\beta}\right) \cap \pi_{\gamma}^{-1}\left(q_{\gamma}\right)\right),
\end{aligned}
$$

because

$$
\pi_{\beta}^{-1}\left(p_{\beta}\right) \cap \pi_{\beta}^{-1}\left(q_{\beta}\right)=\emptyset=\pi_{\gamma}^{-1}\left(p_{\gamma}\right) \cap \pi_{\gamma}^{-1}\left(q_{\gamma}\right),
$$

since by hypothesis, $p_{\beta} \neq q_{\beta}$ and $p_{\gamma} \neq q_{\gamma}$.

Now, by Axiom P2 $\pi_{\alpha}^{-1}\left(x_{\alpha}\right) \in \mathcal{L}$ for all $x \in \prod_{\alpha} \mathcal{L}_{\alpha}$ and all $\alpha \in \Omega$. Therefore,

$$
\left(\pi_{\beta}^{-1}\left(p_{\beta}\right) \cup \pi_{\gamma}^{-1}\left(q_{\gamma}\right)\right) \cap\left(\pi_{\beta}^{-1}\left(q_{\beta}\right) \cup \pi_{\gamma}^{-1}\left(p_{\gamma}\right)\right) \in \mathcal{L} .
$$

As a consequence,

$$
p \vee q \subseteq\left(\pi_{\beta}^{-1}\left(p_{\beta}\right) \cap \pi_{\gamma}^{-1}\left(p_{\gamma}\right)\right) \cup\left(\pi_{\beta}^{-1}\left(q_{\beta}\right) \cap \pi_{\gamma}^{-1}\left(q_{\gamma}\right)\right) .
$$

It follows that either $r_{\beta}=p_{\beta}$ and $r_{\gamma}=p_{\gamma}$, or $r_{\beta}=q_{\beta}$ and $r_{\gamma}=q_{\gamma}$. Since this holds for every pair of indices $\beta, \gamma$ at which $p$ and $q$ differ, either $r=p$ or $r=q$.

(2) Let $\beta \neq \gamma \in \Omega, b \in \mathcal{L}_{\beta}, c \in \mathcal{L}_{\gamma}$, and $p, q \in \boldsymbol{\Sigma}$ such that $p_{\beta} \in b$ and $p_{\gamma} \in c$. We must show that $p[c, \gamma] \cup p[b, \beta] \in \mathcal{L}$. Now, since $p_{\beta} \in b$ and $p_{\gamma} \in c$, we have

$$
\bigcap_{\alpha \neq \beta, \gamma} \pi_{\beta}^{-1}(b) \cap \pi_{\gamma}^{-1}(c) \cap\left(\pi_{\beta}^{-1}\left(p_{\beta}\right) \cup \pi_{\gamma}^{-1}\left(p_{\gamma}\right)\right) \cap \pi_{\alpha}^{-1}\left(p_{\alpha}\right)=p[c, \gamma] \cup p[b, \beta] .
$$

To conclude, it suffices to note that by Axiom P2, the subsets of $\boldsymbol{\Sigma} \pi_{\beta}^{-1}(b), \pi_{\gamma}^{-1}(c)$, $\pi_{\beta}^{-1}\left(p_{\beta}\right) \cup \pi_{\gamma}^{-1}\left(p_{\gamma}\right)$, and $\pi_{\alpha}^{-1}\left(p_{\alpha}\right)$ are elements of $\mathcal{L}$.

Theorem 6.5. Let $\Omega$ be a finite set and $\left\{\mathcal{L}_{i}\right\}_{i \in \Omega}$ a finite family of connected simple closure spaces on $\Sigma_{i}$. Let $\mathcal{L} \in \mathrm{S}\left(\mathcal{L}_{i}, i \in \Omega\right)$ and $u \in \mathbf{C l}(\mathcal{L}, \mathcal{L})$ large, sending atoms to atoms. Then there is a bijection $f$ of $\Omega$, and for each $i \in \Omega$, there is $v_{i} \in$ $\mathbf{C l}\left(\mathcal{L}_{i}, \mathcal{L}_{f(i)}\right)$ sending atoms to atoms such that $u(p)_{f(i)}=v_{i}\left(p_{i}\right)$ for all $p \in \boldsymbol{\Sigma}$ and $i \in \Omega$.

Proof. The proof is similar to the proof of Theorem 3 in 9 .

(1) Let $p \in \boldsymbol{\Sigma}$ and $j \in \Omega$. Claim: There is $k \in \Omega$ such that

$$
u\left(p\left[\Sigma_{j}\right]\right) \subseteq u(p)\left[\Sigma_{k}\right] .
$$

[Proof: Since $\mathcal{L}_{j}$ is connected, for all $q_{j} \in \Sigma_{j}$ different from $p_{j}, p_{j} \vee q_{j}$ contains a third atom, say $r_{j}$, and $p_{j} \in q_{j} \vee r_{j}$ and $q_{j} \in p_{j} \vee r_{j}$. Suppose that $u(p)_{k} \neq u\left(p\left[q_{j}\right]\right)_{k}$ for at least two indices $k$. Then, by Lemma 6.4 part (1) and Lemma 2.7.3,

$$
u\left(p\left[r_{j}\right]\right) \in u\left(p \vee p\left[q_{j}\right]\right)=u(p) \vee u\left(p\left[q_{j}\right]\right)=u(p) \cup u\left(p\left[q_{j}\right]\right) .
$$

Assume for instance that $u\left(p\left[r_{j}\right]\right)=u(p)$. Then,

$$
u\left(p\left[q_{j}\right]\right) \in u\left(p \vee p\left[r_{j}\right]\right)=u(p) \vee u\left(p\left[r_{j}\right]\right)=u(p),
$$


a contradiction, which proves the claim.]

(2) Let $j \in \Omega$. Denote the $k$ of part (1) by $f(j, p)$. Claim: The map $p \mapsto f(j, p)$ is constant. [Proof: Let $p, q \in \boldsymbol{\Sigma}$ differ only by one component, say $j^{\prime} \neq j$, i.e., $p_{j^{\prime}} \neq q_{j^{\prime}}$, and $p_{i}=q_{i}$, for all $i \neq j^{\prime}$. Suppose that $f(j, p) \neq f(j, q)$. Write $k:=f(j, p)$ and $k^{\prime}:=f(j, q)$.

(2.1) We first prove that

$$
u\left(p\left[\Sigma_{j}\right]\right) \vee u\left(q\left[\Sigma_{j}\right]\right)=u\left(p\left[\Sigma_{j}\right]\right) \cup u\left(q\left[\Sigma_{j}\right]\right)
$$

By hypothesis,

$$
u\left(p\left[\Sigma_{j}\right]\right) \subseteq u(p)\left[\Sigma_{k}\right] \text { and } u\left(q\left[\Sigma_{j}\right]\right) \subseteq u(q)\left[\Sigma_{k^{\prime}}\right] .
$$

Hence, for all $r_{j} \in \Sigma_{j}$, we have

$$
u(p)_{l}=u\left(p\left[r_{j}\right]\right)_{l} \forall l \neq k \text { and } u(q)_{m}=u\left(q\left[r_{j}\right]\right)_{m} \forall m \neq k^{\prime} .
$$

Since $u$ is large, $u\left(p\left[\Sigma_{j}\right]\right)$ is not an atom, hence there is $r_{j} \in \Sigma_{j}$ such that

$$
u\left(p\left[r_{j}\right]\right)_{k} \neq u(q)_{k} .
$$

Note that $u(q)_{k}=u\left(q\left[r_{j}\right]\right)_{k}$. Therefore, since $p\left[r_{j}\right]$ and $q\left[r_{j}\right]$ differ only by one component (namely $j^{\prime}$ ), by part (1) we have

$$
u\left(p\left[r_{j}\right]\right)_{l}=u\left(q\left[r_{j}\right]\right)_{l} \forall l \neq k .
$$

As a consequence,

$$
u(q)_{l}=u\left(q\left[r_{j}\right]\right)_{l}=u\left(p\left[r_{j}\right]\right)_{l}=u(p)_{l} \forall l \neq k, k^{\prime},
$$

and

$$
u(p)_{k^{\prime}}=u\left(p\left[r_{j}\right]\right)_{k^{\prime}}=u\left(q\left[r_{j}\right]\right)_{k^{\prime}} \in \pi_{k^{\prime}}\left(u\left(q\left[\Sigma_{j}\right]\right)\right) .
$$

On the other hand, since $u\left(q\left[\Sigma_{j}\right]\right)$ is not an atom, there is $s_{j} \in \Sigma_{j}$ such that

$$
u\left(q\left[s_{j}\right]\right)_{k^{\prime}} \neq u(p)_{k^{\prime}} .
$$

Note that $u(p)_{k^{\prime}}=u\left(p\left[s_{j}\right]\right)_{k^{\prime}}$. Therefore, since $q\left[s_{j}\right]$ and $p\left[s_{j}\right]$ differ only by on component (namely $j^{\prime}$ ), by part (1) we have

$$
u\left(q\left[s_{j}\right]\right)_{l}=u\left(p\left[s_{j}\right]\right)_{l} \forall l \neq k^{\prime} .
$$

As a consequence,

$$
u(q)_{k}=u\left(q\left[s_{j}\right]\right)_{k}=u\left(p\left[s_{j}\right]\right)_{k} \in \pi_{k}\left(u\left(p\left[\Sigma_{j}\right]\right)\right) .
$$

To summarize, we have proved that $u(p)_{l}=u(q)_{l}$ for all $l \neq k, k^{\prime}, u(p)_{k^{\prime}} \in$ $\pi_{k^{\prime}}\left(u\left(q\left[\Sigma_{j}\right]\right)\right)$, and $u(q)_{k} \in \pi_{k}\left(u\left(p\left[\Sigma_{j}\right]\right)\right)$. As a consequence, the statement follows from Lemma 6.4 part (2).

(2.2) Since $\mathcal{L}_{j^{\prime}}$ is connected, there is $s_{j^{\prime}} \in p_{j^{\prime}} \vee q_{j^{\prime}}$ such that $p_{j^{\prime}} \in q_{j^{\prime}} \vee s_{j^{\prime}}$ and $q_{j^{\prime}} \in p_{j^{\prime}} \vee s_{j^{\prime}}$. Let $r=p\left[s_{j^{\prime}}\right]$. Then, by Lemma 2.7] 3 ,

$$
r\left[\Sigma_{j}\right] \subseteq p\left[\Sigma_{j}\right] \vee q\left[\Sigma_{j}\right], \quad p\left[\Sigma_{j}\right] \subseteq q\left[\Sigma_{j}\right] \vee r\left[\Sigma_{j}\right] \quad \text { and } \quad q\left[\Sigma_{j}\right] \subseteq p\left[\Sigma_{j}\right] \vee r\left[\Sigma_{j}\right] .
$$

Now, by part (2.1),

$$
u\left(p\left[\Sigma_{j}\right] \vee q\left[\Sigma_{j}\right]\right)=u\left(p\left[\Sigma_{j}\right]\right) \vee u\left(q\left[\Sigma_{j}\right]\right)=u\left(p\left[\Sigma_{j}\right]\right) \cup u\left(q\left[\Sigma_{j}\right]\right) .
$$

As a consequence, by part $(1), u\left(r\left[\Sigma_{j}\right]\right) \subseteq u\left(p\left[\Sigma_{j}\right]\right)$ or $u\left(r\left[\Sigma_{j}\right]\right) \subseteq u\left(q\left[\Sigma_{j}\right]\right)$. Assume for instance that $u\left(r\left[\Sigma_{j}\right]\right) \subseteq u\left(p\left[\Sigma_{j}\right]\right)$. Then,

$$
u\left(q\left[\Sigma_{j}\right]\right) \subseteq u\left(p\left[\Sigma_{j}\right] \vee r\left[\Sigma_{j}\right]\right)=u\left(p\left[\Sigma_{j}\right]\right) \vee u\left(r\left[\Sigma_{j}\right]\right)=u\left(p\left[\Sigma_{j}\right]\right),
$$

a contradiction. Hence, we have proved that if $p, q \in \boldsymbol{\Sigma}$ differ only by one component, then $f(j, p)=f(j, q)$. 
(2.3) Suppose now that $p$ and $q$ differ by more than one component. Since $\Omega$ is finite, there is $s_{1}, \cdots, s_{n} \in \boldsymbol{\Sigma}$ such that $s^{1}=q, s^{n}=p$, and such that for all $1 \leq i \leq n-1, s^{i}$ and $s^{i+1}$ differ only by one component. Therefore,

$$
f(j, q)=f\left(j, s^{1}\right)=f\left(j, s^{2}\right)=\cdots=f\left(j, s^{n}\right)=f(j, p),
$$

and we are done.]

(3) Let $p_{0} \in \boldsymbol{\Sigma}$. Define $f: \Omega \rightarrow \Omega$ as $f(i):=f\left(i, p_{0}\right)$. Note that by part $(2)$, $f$ does not depend on the choice of $p_{0}$. Claim: The map $f$ is surjective. [Proof: Let $k \in \Omega$. Suppose that for all $i \in \Omega f(i) \neq k$. Let $p, q \in \boldsymbol{\Sigma}$ that differ only by one component, say $j$. Then $p\left[\Sigma_{j}\right]=q\left[\Sigma_{j}\right]$, therefore $u\left(p\left[\Sigma_{j}\right]\right)=u\left(q\left[\Sigma_{j}\right]\right)$. Moreover, by part (1), there is $k^{\prime} \neq k$ such $u\left(p\left[\Sigma_{j}\right]\right) \subseteq u(p)\left[\Sigma_{k^{\prime}}\right]$. As a consequence, $u(p)_{k}=u(q)_{k}$.

Since $\Omega$ is finite, by the same argument as in part $(2.3)$, we find that $u(p)_{k}=$ $u(q)_{k}$, for all $p, q \in \boldsymbol{\Sigma}$. As a consequence, $u(\boldsymbol{\Sigma}) \subseteq \pi_{k}^{-1}\left(u\left(p_{0}\right)_{k}\right)$, a contradiction since $u$ is large.]

(4) Let $p_{0} \in \boldsymbol{\Sigma}$ and $j \in \Omega$. Define $v_{j}: \mathcal{L}_{j} \rightarrow \mathcal{L}_{f(j)}$ as

$$
v_{j}\left(a_{j}\right):=\pi_{f(j)}\left(u\left(p_{0}\left[a_{j}, j\right]\right)\right) .
$$

Claim: $v_{j}$ does not depend on the choice of $p_{0}$. [Proof: Let $q \in \boldsymbol{\Sigma}$ that differs from $p_{0}$ only by one component, say $j^{\prime} \neq j$. Then, by Lemma 2.7.3, we have

$$
\begin{aligned}
\pi_{f(j)}\left(u\left(q\left[a_{j}\right]\right)\right) & =\pi_{f(j)}\left(u\left(\vee\left\{q\left[r_{j}\right] ; r_{j} \in a_{j}\right\}\right)\right) \\
& =\pi_{f(j)}\left(\vee\left\{u\left(q\left[r_{j}\right]\right) ; r_{j} \in a_{j}\right\}\right)=\bigvee_{r_{j} \in a_{j}} u\left(q\left[r_{j}\right]\right)_{f(j)},
\end{aligned}
$$

and the same formula holds for $\pi_{f(j)}\left(u\left(p_{0}\left[a_{j}\right]\right)\right)$. Now

$$
u\left(q\left[r_{j}\right]\right)_{f(j)}=\pi_{f(j)}\left(u\left(s\left[\Sigma_{j^{\prime}}\right]\right)\right)=u\left(p_{0}\left[r_{j}\right]\right)_{f(j)},
$$

where $s=q\left[r_{j}\right]$. As a consequence, $\pi_{f(j)}\left(u\left(q\left[a_{j}\right]\right)\right)=\pi_{f(j)}\left(u\left(p_{0}\left[a_{j}\right]\right)\right)$.

Since $\Omega$ is finite, by the same argument as in part $(2.3)$, we find that

$$
\pi_{f(j)}\left(u\left(q\left[a_{j}\right]\right)\right)=\pi_{f(j)}\left(u\left(p_{0}\left[a_{j}\right]\right)\right)
$$

for all $q \in \boldsymbol{\Sigma}$.]

It remains to check that $v_{j}$ preserves arbitrary joins. Let $\omega \subseteq \mathcal{L}_{j}$. Then, by Lemma 2.7]3,

$$
\begin{aligned}
v_{j}(\bigvee \omega) & =\pi_{f(j)}\left(u\left(p_{0}[\vee \omega]\right)\right)=\pi_{f(j)}\left(u\left(\vee\left\{p_{0}[x, j] ; x \in \omega\right\}\right)\right) \\
& =\pi_{f(j)}\left(\vee\left\{u\left(p_{0}[x, j]\right) ; x \in \omega\right\}\right) \\
& =\bigvee_{x \in \omega} \pi_{f(j)}\left(u\left(p_{0}[x, j]\right)\right)=\bigvee_{x \in \omega} v_{j}(x) .
\end{aligned}
$$

Corollary 6.6. If the $u$ in Theorem 6.5 is an automorphism, then all $v_{i}$ 's are isomorphisms.

Theorem 6.7. If the $u$ in Theorem 6.5 is an automorphism, the statement remains true if for all $i \in \Omega, \mathcal{L}_{i}$ is weakly connected.

Proof. The proof is similar as in Theorem 6.5. We only sketch the arguments that must be modified. Note that since $u$ is an automorphism and $\mathcal{L}_{i} \neq 2$ for all $i \in \Omega$, $u$ is large. 
(1) Since $\mathcal{L}_{j}$ is weakly connected, there is $\gamma_{0} \in \sigma^{j}$ such that $p_{j} \in A_{j}^{\gamma_{0}}$. By hypothesis and Lemma 2.7.3, for all $q_{j} \in A_{j}^{\gamma_{0}}, p \vee\left(p\left[q_{j}\right]\right)$ contains a third atom, hence also $u(p) \vee u\left(p\left[q_{j}\right]\right)$ since $u$ is injective. As a consequence, there is $k_{\gamma_{0}} \in \Omega$ such that $u\left(p\left[A_{j}^{\gamma_{0}}\right]\right) \subseteq u(p)\left[\Sigma_{k_{\gamma_{0}}}\right]$. Moreover, since $u$ is injective, by the third hypothesis in Definition 6.1 the map $\gamma \mapsto k_{\gamma}$ is constant. Therefore, since $\cup A_{j}^{\gamma}=\Sigma_{j}$, we find that $u\left(p\left[\Sigma_{j}\right]\right) \subseteq u(p)\left[\Sigma_{k}\right]$.

(2) Take $p, q \in \boldsymbol{\Sigma}$ that differ only by one component such that $q_{j^{\prime}}$ and $p_{j^{\prime}}$ are in the same $A_{j}^{\gamma}$.

(2.2) By hypothesis, $p_{j^{\prime}} \vee q_{j^{\prime}}$ contains a third atom, say $r_{j^{\prime}}$, therefore $r\left[\Sigma_{j}\right] \subseteq$ $p\left[\Sigma_{j}\right] \vee q\left[\Sigma_{j}\right]$, hence, $u\left(r\left[\Sigma_{j}\right]\right)=u\left(p\left[\Sigma_{j}\right]\right)$ or $u\left(r\left[\Sigma_{j}\right]\right)=u\left(q\left[\Sigma_{j}\right]\right)$, a contradiction since $u$ is injective. As a consequence, $f(j, q)=f(j, p)$. Now, since $u$ is injective, by the third hypothesis in definition 6.1 we find that $f(j, q)=f(j, p)$, for all $p, q \in \boldsymbol{\Sigma}$ that differ only by the component $j^{\prime}$.

\section{ACKNOWLEDGMENTS}

A part of this work was done during a stay at McGill. In this connection, I would like to thanks M. Barr for his hospitality.

\section{REFERENCES}

[1] D. Aerts. Description of many separated physical entities without the paradoxes encountered in quantum mechanics. Found. Phys., 12(12):1131-1170, 1982.

[2] M. Barr. *-autonomous categories, volume 752 of Lecture Notes in Mathematics. Springer, Berlin, 1979. With an appendix by Po Hsiang Chu.

[3] G. Birkhoff and J. von Neumann. The logic of quantum mechanics. Ann. of Math. (2), 37(4):823-843, 1936.

[4] C.-A. Faure, D. J. Moore, and C. Piron. Deterministic evolutions and Schrödinger flows. Helv. Phys. Acta, 68(2):150-157, 1995.

[5] G. A. Fraser. The semilattice tensor product of distributive lattices. Trans. Amer. Math. Soc., 217:183-194, 1976

[6] A. S. Golfin. Representations and products of lattices. PhD thesis, U. Mass., Amherst, 1987.

[7] G. Grätzer and F. Wehrung. A new lattice construction: the box product. J. Algebra, 221(1):315-344, 1999.

[8] B. Ischi. Orthocomplementation and compound systems. Internat. J. Theoret. Phys., 44(12):2207-2217, 2005.

[9] B. Ischi. Endomorphisms of the separated product of lattices. Internat. J. Theoret. Phys., 39(11):2559-2581, 2000.

[10] F. Maeda and S. Maeda. Theory of symmetric lattices. Die Grundlehren der mathematischen Wissenschaften, Band 173. Springer-Verlag, New York, 1970.

[11] G. J. Seal. Cartesian closed topological categories and tensor products. Appl. Categ. Structures, 13(1):37-37, 2005.

[12] Z. Shmuely. The structure of Galois connections. Pacific J. Math., 54(2):209-225, 1974.

Boris Ischi, Laboratoire de Physique des Solides, Université Paris-Sud, Bâtiment 510, 91405 Orsay, France

Current address: Collège de Candolle, 5 rue d'Italie, 1204 Geneva, Switzerland

E-mail address: boris.ischi@edu.ge.ch 